\title{
Macrophages in tumor-associated adipose microenvironment accelerate tumor progression
}

Bei Li

Wuhan University Renmin Hospital

Qi Wu

Wuhan University Renmin Hospital

Qian Yang

Wuhan University Renmin Hospital

Zhiyu Li

Wuhan University Renmin Hospital

Juanjuan Li

Wuhan University Renmin Hospital

Juan Wu

Wuhan University Renmin Hospital

Shan Zhu

Wuhan University Renmin Hospital

Lijun Wang

Wuhan University Renmin Hospital

Shichong Liao

Wuhan University Renmin Hospital

Si Sun

Wuhan University Renmin Hospital

Zhiliang Xu

Wuhan University Renmin Hospital

Shengrong Sun ( $\nabla$ sun137@sina.com )

Renmin Hospital of Wuhan University https://orcid.org/0000-0003-2893-6735

Research

Keywords: Exosome, cancer, adipocyte, macrophage, obesity

Posted Date: May 6th, 2021

DOI: https://doi.org/10.21203/rs.3.rs-86067/v3 
License: (c) (i) This work is licensed under a Creative Commons Attribution 4.0 International License. Read Full License 


\section{Abstract}

Background: Macrophages particularly contribute to the progression of obesity-related tumor. However, the mechanisms that the tumor-adipocyte crosstalk may enable the properties and plasticity of macrophages remain still unclear.

Methods: Survival probabilities for recurrence-free survival (RFS) were estimated by the Kaplan-Meier method based on immunohistochemistry and immunofluorescence images. 3T3-L1 cells were cocultivated with $4 \mathrm{~T} 1$ and MDA-MB-231 cells. Then the co-cultivated media were used to treat THP1/RAW264.7 cells. The markers of macrophages were detected by immunofluorescence and Western blot. Transwell migration assay, was conducted to determine the migration capability of macrophages. RT-PCR and ELISA were used to detect the expression and secretion level of chemokines, respectively. Immunofluorescence imaging and Western blot were used to investigate the role of SOCS6/STAT3 signaling pathway in the polarization of macrophages. microRNA mimic and inhibitor, and xenograft models were used to explore the role of miR-155 in the polarization of macrophages.

Results: We demonstrate that CD163-positive macrophages aggregated to surround adipocytes in breast cancer tissues, which was associated with tumor relapse. In tumor-adipocyte microenvironment, CD163positive macrophages are recruited and polarized via the elevated expression of CCL2 and CCL5. Consistently, the eliminated macrophages partially inhibited adipocytes-induced tumor proliferation. Likewise, inhibiting chemokines and their receptors or suppressing the phosphorylation of STAT3 significantly decreased tumor burden in vivo. Finally, the source of CCL2 and CCL5 mainly derived from adipocytes. In coculture of tumor cells and adipocytes, the level of exosomal miRNA-155 was high, then it promoted the generation and release of CCL2 and CCL5 from adipocytes through targeting SOCS6/STAT3 pathway. Inhibition of exosomal miRNA-155 in tumor cells reduced the CCL2 and CCL5 levels in tumor-adipocytes coculture, and further retarded tumor growth.

Conclusion: These results suggest a novel target of tumor-adipocyte-macrophage interconnect that could facilitate obesity-induced tumor progression.

\section{Introduction}

Obesity is a growing global epidemic and results in preventable risk factor for cancer incidence and mortality, being responsible for an estimated $\sim 20 \%$ of cancer-related deaths in adults[1]. Obesity is mainly dependent on excessive fat accumulation in the regional adipose tissues. However, growing evidence from both clinical and preclinical studies indicates that increased adiposity is associated with increases in cancer incidence and mortality [2,3], mechanistic insights explaining these data are notably lacking. In particular, obesity-caused chronic inflammation is considered as a potent driver of cancer initiation and progression through its ability to cultivate a supported microenvironment that is permissive to neoplastic transformation[4-6]. 
Macrophages, key cells in the inflammatory response, also enrich in adipose and tumor microenvironments, where they mediate the development, growth, and progression of cancer [7]. Macrophages in adipose and tumor microenvironments are a diverse population of cells that develop from different origins and express distinct surface markers[8]. Tissue-resident macrophages like adiposetissue macrophages (ATMs) are originated from embryonic precursors or adult haematopoietic stem cells (HSCs). In the inflammatory environments, a relevant proportion of macrophages also derive from monocyte-derived cells. Classically, two main phenotypes of macrophages in adipose tissue are proinflammatory M1 (classically activated) and anti-inflammatory M2 (alternatively activated) [9]. The macrophages in lean adipose tissue are polarized toward the M2 activation state, whereas in obese adipose tissue, they are toward the M1 activation state [10]. M1 macrophages produce pro-inflammatory cytokines (TNF-a, interleukin IL-6) while M2 macrophages play an anti-inflammatory role via secreting IL10[11]. Moreover, macrophage phenotype can vary in different tumor types and intratumor districts[11]. Specifically, macrophages can be polarized into an M2-like state or "switching macrophages", which produce both pro- and anti-inflammatory cytokines and have been observed in tumors and adipose tissues[12,13]. Interestingly, the macrophages that envelope around phagocytosis of a dead or dying adipocyte in a configuration termed crown-like structures (CLSs), a histologic biomarker of inflammation [2]. In breast cancer patients, the pathologically increase of CLSs in mammary adipose tissue is related to poor prognosis, estrogen receptor negativity and the overexpression of positive human epidermal growth factor receptor-2[14, 15]. Hence, macrophage collection in adipose tissue nearby the breast cancer lesion appears to have important clinical implications, particularly regarding macrophage properties; however, it has not been thoroughly investigated.

Chemokines are discovered as a family of secreted proteins, whose prominent role is leukocyte chemotaxis and activation[16]. Chemokine (C-C motif) ligand 2 (CCL2) and CCL5 are a member of chemokine branch of the cytokine superfamily. CCL2 attracts circulating macrophages and stimulates their immunosuppression in the tumor microenvironment via binding to its receptor CCR-2[17].

Meanwhile, CCL5 could recruit CCR5-overexpressing macrophages, subsequently which depose collagens in residual tumors to drive tumor recurrence[18]. However, whether the expressions of CCL2 and CCL5 are elevated in tumor-associated adipose microenvironment, and repolarize the residual macrophages is less well characterized.

Exosomes are a kind of small extracellular vesicles $(30-100 \mathrm{~nm})$ derived from cancer cells and stromal cells[19]. This cell-to-cell biological communication is mediated through the exchange of the exosomes content, which consists of proteins, lipids, metabolites, RNA, and DNA [20]. Exosomes are appreciated as essential mediators of cell-cell communication. Our previous research indicated that exosomal miRNAs from the tumour-adipocyte interaction induce beige/brown differentiation and accelerate catabolism in recipient adipocytes to facilitate tumour progression[21-23]. In this study, it's evident that CCL2 and CCL5 facilitate tumor growth through recruiting and polarizing M2-like macrophages in tumor-adipose microenvironment. In addition, the results also show that exosomes derived from tumor-adipocyte interaction induce CCL2 and CCL5 secretion from adipocytes in SOCS6/STAT3-depending way. 


\section{Material And Methods Patients}

A total of 145 formalin-fixed paraffin-embedded (FFPE) tissue samples of breast cancer were obtained from Renmin Hospital of Wuhan University. All patients involved in the study provided a written informed consent, and the study was approved by the Institutional Ethics Committee of Renmin Hospital of Wuhan University (approval no. 2018K-C09). Patients did not receive financial compensation. Clinical information was extracted from medical records and pathology reports, and the detailed clinicopathological characteristics of the patient are shown in Table 1. Patients were all followed-up for at least 5 years from the date of first diagnosis. All methods were performed in accordance with relevant guidelines and local regulations. 
Table 1

Clinicopathological associations of $\mathrm{CD}_{163^{+}}$macrophages in breast cancer.

\begin{tabular}{|c|c|c|c|}
\hline $\begin{array}{l}\text { Clinicopathological } \\
\text { parameters }\end{array}$ & CD163 Negative & CD163 Positive & $P$ value \\
\hline Age at diagnosis, y & & & 0.688 \\
\hline$\leq 50$ & $35(50.0)$ & $35(46.7)$ & \\
\hline$\geq 51$ & $35(50.0)$ & $40(53.3)$ & \\
\hline Tumor size(cm) & & & 0.017 \\
\hline$<2$ & $34(48.6)$ & $22(29.3)$ & \\
\hline$\geq 2$ & $36(51.4)$ & $53(70.7)$ & \\
\hline Lymph node metastasis & & & 0.000 \\
\hline Negative & $45(64.3)$ & $26(34.7)$ & \\
\hline Positive & $25(35.7)$ & $49(65.3)$ & \\
\hline Vascular invasion & & & 0.121 \\
\hline Negative & $66(94.3)$ & $65(86.7)$ & \\
\hline Positive & $4(5.7)$ & $10(13.3)$ & \\
\hline ER & & & 0.216 \\
\hline Negative & $32(45.7)$ & $42(56.0)$ & \\
\hline Positive & $38(54.3)$ & $33(44.0)$ & \\
\hline PR & & & 0.123 \\
\hline Negative & $34(48.6)$ & $46(61.3)$ & \\
\hline Positive & $36(51.4)$ & $29(38.7)$ & \\
\hline HER2 & & & 0.414 \\
\hline Negative & $51(72.9)$ & $59(78.7)$ & \\
\hline Positive & 19(27.1) & 16(21.3) & \\
\hline Ki67 & & & 0.007 \\
\hline$<14 \%$ & $40(57.1)$ & $26(34.7)$ & \\
\hline$\geq 14 \%$ & $30(42.9)$ & 49(65.3) & \\
\hline
\end{tabular}

*P values calculated by Log-rank testing; Bold if statistically significant, $P<0.05$. ER: estrogen receptor, PR: progesterone receptor, HER2: human epithelial growth factor receptor-2. 


\begin{tabular}{|c|c|c|c|}
\hline $\begin{array}{l}\text { Clinicopathological } \\
\text { parameters }\end{array}$ & CD163 Negative & CD163 Positive & $P$ value \\
\hline Recurrence & & & 0.007 \\
\hline No & $54(77.1)$ & $42(56.0)$ & \\
\hline Yes & $16(22.9)$ & $33(44.0)$ & \\
\hline
\end{tabular}

\section{Cell culture and reagents}

The breast cancer cell lines AT3, 4T1, MCF-7, MDA-MB-231 and monocyte lines Raw264.7, THP-1 cells were obtained from American Type Culture Collection (ATCC, Shanghai). The mouse breast cancer cell line 4T1 and human monocyte line THP-1 were cultured in Roswell Park Memorial Institute (RMPI)-1640 medium supplemented with $10 \%$ foetal bovine serum (FBS, AusGeneX) and $1 \%$ penicillin-streptomycin (HyClone, Logan, UT, USA) in a humidified $37^{\circ} \mathrm{C}$ incubator with $5 \% \mathrm{CO} 2$. The human breast cancer cell lines MCF-7, MDA-MB-231 and mouse macrophage line Raw264.7 were cultured in Dulbecco's modified Eagle's medium (DMEM) supplemented with $10 \%$ FBS and $1 \%$ penicillin-streptomycin in a humidified $37^{\circ} \mathrm{C}$ incubator with $5 \% \mathrm{CO} 2.3 \mathrm{~T} 3-\mathrm{L} 1$ preadipocytes were obtained from ATCC (Shanghai) and cultured in DMEM supplemented with $10 \%$ FBS and $1 \%$ penicillin-streptomycin in a humidified $37^{\circ} \mathrm{C}$ incubator with $5 \%$ CO2. Cytochalasin D, Bafilomycin A1(Baf-A1) and insulin were purchased from Sigma.

\section{Coculture and invasion assays}

Mature 3T3-L1 and breast cancer cells were cocultured using Transwell culture plates $(0.4 \mu \mathrm{m}$ pore size; Millipore). Mature 3T3-L1 cells in the bottom chamber of the Transwell system were cultivated in serumfree medium containing $1 \%$ bovine serum albumin (Sigma) for $4 \mathrm{~h}$. A total of $3 \times 10^{\wedge} 54 \mathrm{~T} 1$ or MCF-7 or MDA-MB-231 cells were cultivated in the top chamber in the presence or absence of mature 3T3-L1 cells in the bottom chamber for the indicated times. The conditioned medium (CA-CM) was collected from adipocytes cultivated with tumour cells for 3 days. After $24 \mathrm{~h}$ of coculture in the presence of normal medium or CA-CM (supplemented with $10 \%$ FBS), macrophages were seeded in the top chamber in the presence of $\mathrm{CM}, \mathrm{AD}-\mathrm{CM}$ or CA-CM (supplemented with $10 \%$ FBS) for Matrigel invasion assays.

\section{THP-1 Differentiation}

A total of $5 \times 10^{\wedge} 6$ THP-1 cells were seeded in a 6-well plate with Phorbol 12-myristate 13-acetate (PMA, 100ng/ml, MedChemExpress, 16561-29-8) supplemented with 1\% FBS in RMPI-1640. After 24h, RMPI1640 medium with PMA was replaced by normal medium for 2 days.

\section{Exosome isolation and characterization}

After cells were cultured with exosome-depleted serum (AusGeneX), the exosomes were purified from the conditioned medium according to the instructions[24]. The medium was centrifuged at $500 \mathrm{~g}$ for $5 \mathrm{~min}$ 
and at $2000 \mathrm{~g}$ for $30 \mathrm{~min}$ at $4^{\circ} \mathrm{C}$ to remove cellular debris and large apoptotic bodies. After centrifugation, media was added to an equal volume of a $2 \times$ polyethylene glycol (PEG, MW 6000, Sigma, 81260) solution (final concentration, $8 \%$ ). The samples were mixed thoroughly by inversion and incubated at $4^{\circ} \mathrm{C}$ overnight. Before the tubes were tapped occasionally and drained for $5 \mathrm{~min}$ to remove excess PEG, the samples were further centrifuged at maximum speed $(15,000 \mathrm{rpm})$ for $1 \mathrm{~h}$ at $4^{\circ} \mathrm{C}$. The resulting pellets were further purified using 5\% PEG and then stored in $50-100 \mu \mathrm{l}$ of particle-free PBS $(\mathrm{pH} 7.4)$ at $-80^{\circ} \mathrm{C}$. The average yield was approximately $300 \mu \mathrm{g}$ of exosomal protein from $5 \mathrm{ml}$ of supernatant. Total RNA was extracted by using Trizol reagent (Life Technologies), followed by miRNA assessment by microarrays and RT-PCR described below. Exosomes were analyzed by electron microscopy to verify their presence, by a nanoparticle characterization system to measure their size and concentration, and by western blot to detect their proteins (TSG101, CD63 and CD81).

\section{Electron microscopy}

After being fixed with $2 \%$ paraformaldehyde, samples were adsorbed onto nickel formvar-carbon-coated electron microscopy grids (200 mesh), dried at room temperature, and stained with $0.4 \%(\mathrm{w} / \mathrm{v})$ uranyl acetate on ice for 10 min. The grids were observed under a HITACHI HT7700 transmission electron microscope.

\section{Nanoparticle characterization system (NanoSight)}

The NanoSight (Malvern Zetasizer Nano ZS-90) was used for real-time characterization and quantification of exosomes in PBS as specified by the manufacturer's instructions.

\section{Exosome uptake analysis}

Exosomes derived from breast cancer cells were labeled by the cell membrane labeling agent PKH26 (Sigma-Aldrich). After being seeded in 96-well plates and allowed to differentiate, mature 3T3-L1 cells were incubated with labelled exosomes $(20 \mu \mathrm{l} /$ well) for the indicated time. Images were acquired using the Olympus FluoView FV1000.

\section{ELISA}

The conditioned media were collected to analyze the secretion levels of CCL2 and CCL5 via Mouse MCP1/CCL2 ELISA kit (Sizhengbai, CME0046) and Mouse CCL5/RANTES ELISA kit (Sizhengbai, CME0048). The conditioned media were centrifuged at $1000 \mathrm{~g}$ for $10 \mathrm{~min}$, incubated with biotinylated antibody working solution at $37^{\circ} \mathrm{C}$ for $1.5 \mathrm{~h}$, incubated with enzyme conjugated working solution at $37^{\circ} \mathrm{C}$ for $0.5 \mathrm{~h}$, and color developing for $10 \mathrm{~min}$.

\section{Western blotting}

After being washed twice with ice-cold PBS, cells were collected with SDS loading buffer and boiled for $10 \mathrm{~min}$. The proteins were separated by SDS-PAGE, transferred to a nitrocellulose membrane, and detected with specific antibodies (Additional file 1: Table S1). 


\section{Neutralizing experiments}

Before used to treat macrophages, CA-CM was neutralized by goat serum or CCL2 or CCL 5 neutralizing antibodies overnight. Macrophages were treated with the neutralized CA-CM for 3 days, and then were collected for western blot analysis.

\section{RNA extraction and quantitative PCR}

Gene expression was analyzed using real-time PCR. The mRNA primer sequences are provided in Additional file 1: Table S2. The miRNA primer kits were purchased from RiboBio (Guang Zhou, China).

\section{Immunohistochemistry}

A cohort of 145 human breast cancer specimens was collected from Renmin Hospital of Wuhan University from 2011 to 2013. Immunohistochemistry (IHC) staining was performed, and the staining results were scored by two independent pathologists based on the proportion of positively stained tumour cells and the staining intensity. The protein expression level of CD163 was described according to the numbers of CD163 + macrophages counted in 10 random fields of each breast cancer specimen at 400x magnification. The intensity of protein expression was scored as 0 (no staining), 1 (weak staining, light brown), 2 (moderate staining, brown) and 3 (strong staining, dark brown). The protein staining score was determined using the following formula: overall score $=$ percentage score $\times$ intensity score. Receiver operating characteristic (ROC) analysis was used to determine the optimal cut-off values for all expression levels regarding the survival rate.

\section{Immunofluorescence imaging}

Immunofluorescence (IF) imaging was performed to investigate the localization of pSTAT3 (Tyr705) (\#9145, 1:200, Cell Signaling Technology), CD68 and CD163. Tissue specimens undergoing IF staining were incubated with Alexa Fluor -conjugated secondary antibodies against the primary antibodies for $1 \mathrm{~h}$ at room temperature, followed by counterstaining with DAPI for $5 \mathrm{~min}$. Images were captured using a fluorescence microscope (Olympus BX63; Olympus Corporation).

\section{Image segmentation and data analysis}

Images were segmented using the EBImage package (available from Bioconductor repository https://www.bioconductor.org) with the $\mathrm{R}$ software. The nuclear region was defined using a polygon mask based on the nuclear Hoechst signal, and a second polygon mask was generated using the GFP or RFP signal. For the assessment of autophagic vesicles, a third mask was created on cytoplasmic regions exhibiting a high intensity signal of GFP corresponding with LC3 aggregates.

\section{Luciferase assays}

The 3' UTRs of target genes containing predicted miRNA binding sites (gene ${ }^{\mathrm{wt}}$ ) were cloned into the GV272 vector (GeneChem Biotechnology, Shanghai, China), and the miRNA binding sites were replaced with a 4-nt fragment to produce a mutated 3' UTR (gene ${ }^{\text {mut }}$ ) in the vector. Briefly, HEK 293 T cells were 
seeded in 12-well plates and grew to $70 \%$ confluence. The cells were transfected with gene ${ }^{\text {wt }}$ or gene ${ }^{\text {mut }}$, the pre-miRNA expression plasmid and pRL-SV40, which constitutively expresses Renilla luciferase as an internal control. At $48 \mathrm{~h}$ post-transfection, the cells were lysed, and Renilla luciferase activity was assessed by the TECAN Infiniti reader. The results are described as the ratio of firefly luciferase activity compared to Renilla luciferase activity.

\section{Lentivirus preparation and transfection}

siRNA-TFE3 (5'-GGAAUCUGCUUGAUGUGUA-3') (GE Healthcare, Dharmacon) were used. LentiBrite GFPLC3 lentiviral biosensor was purchased from MERCK (\#17-10193, Darmstadt, Germany). miRNA-155 inhibitors and pre-miRNA lentivirus were obtained from GeneChem Biotechnology (Shanghai, China). Cells were cultured at $5 \times 105$ cells/well in 6-well plates. After being incubated for $24 \mathrm{~h}$, the cells were transfected with siRNA lentivirus and control sequences using CON036 (GeneChem Biotechnology, China) following the manufacturer's instructions. Cells $(2 \times 105)$ were stably transfected with empty vector or with vectors carrying miRNA inhibitor or pre-miRNA using the TransIT-LT1 reagent (Mirus). Selection was carried out with puromycin $(1 \mu \mathrm{g} / \mathrm{ml}$, Sigma) or G418 $(500 \mu \mathrm{g} / \mathrm{ml}$, Sigma) in cell culture media for $48 \mathrm{~h}$ after transfection. Selected clones were maintained in DMEM with $500 \mu \mathrm{g} / \mathrm{ml} \mathrm{G} 418$ or $1 \mu \mathrm{g} / \mathrm{ml}$ puromycin. Cell lysates were collected, and RT-PCR was performed to detect miRNA expression. The sequence information is provided in Additional file 3: Table S3.

\section{Xenograft tumor formation}

Six-week-old female BALB/c mice were purchased from Vital River, Beijing. The animals were handled according to the protocol approved by the Institutional Animal Care and Use Committee of Renmin Hospital of Wuhan University. The following cell lines were used to create subcutaneous models: $4 \times 10^{5}$ 4T1 cells treated with control lentivirus, transfected with miRNA-155 inhibitor lentivirus. Breast cancer cells were injected alone or in combination with mature adipocytes $\left(1 \times 10^{5}\right.$ cells). All cell samples were injected with Matrigel (1:1), total volume $100 \mu$, into the mammary fat pad or the axilla of the mice. For macrophages deletion, mice were treated with blocking antibodies against F4/80 as described.

Six-week-old female wild-type C57BL/6 mice were obtained from Vital River, Beijing. Mouse Mammary Carcinoma AT-3 cells $\left(2 \times 10^{5}\right)$ alone or in combination with mature adipocytes $\left(1 \times 10^{5}\right.$ cells) were injected into C57BL/ 6 hosts under the mammary gland fat. When tumors became palpable, mice were treated with the STAT3 inhibitor Stattic (10 mg/kg, days 0 and 7, Sigma-Aldrich), blocking antibodies against CCL2 (BioXcell, West Lebanon, NH, USA) and/or CCL5 (200 $\mu$ g, days - 1 and 0, and were repeated every $3 \mathrm{~d}$ thereafter to maintain depletion or neutralization) (R\&D Systems, Minnesota, USA) by intraperitoneal injection, or the CCR2/CCR5 antagonist BMS-813160 (10 mg/kg, gavage, days 0, 4, 8 and 12) (MedChemEcpress, Shanghai, China). On the following days, mice well-being and tumor growth were monitored and documented. Tumour volume was defined as (longest diameter) $\times$ (shortest diameter) ${ }^{2}$ $\times 0.52$ and was measured once every $2-3$ days until using a Vernier calliper. Animals were sacrificed when tumor size reached endpoint or signs of obvious discomfort were observed following our Ethical 
Committee advice. After the mice were sacrificed, all tissues were collected, embedded in paraffin and stained with IHC or haematoxylin-eosin (HE).

\section{Statistical analysis}

All experiments were done independently at least three times. The results are presented as the mean \pm SD. The relative increase in protein expression was quantified using Image $\mathrm{J}$ software and was normalized to control protein expression in each experiment. Data sets obtained from different experimental conditions were compared with the t-test when comparing only 2 groups. Multiple comparisons between groups were performed using the Mann-Whitney U test or Tukey's multiple comparisons. Survival probabilities for recurrence-free survival (RFS) were estimated using the Kaplan-Meier method, and variables were compared using the log-rank test. Pearson's correlation was used to evaluate the correlations among CD163, CCL2 and CCL5 expression levels. In the bar graphs, a single asterisk (*) indicates $\mathrm{P}<0.05$.

\section{Results}

The infiltration of M2-like macrophages in tumor-surrounding adipose tissues were associated with poor prognosis in patients with breast cancer

The heterogeneity and plasticity of macrophages in adipose tissues surrounding by breast cancer has not been thoroughly explored. It is reported that tumor-associated macrophages (TAMs) are confirmed as "switching macrophages", which generate pro- and anti-inflammatory cytokines meantime and are found in tumors[13]. CD68 (macrophage membrane marker) and CD163 (M2-macrophage marker) have been usually regarded as surrogate markers to investigate TAMs polarity in previous studies $[15,25]$. And the increased infiltration of $\mathrm{CD}_{163^{+}}$tumor-associated macrophages (TAMs) in CLSs were associated with shorter disease-free survival in node-negative breast cancer patients[26]. We firstly detected the existence and characteristics of macrophages in a cohort of 145 breast cancer specimens using immunohistochemistry (IHC) and QDs-based fluorescent imaging technique (Table 1). We detected the expression of CD68 and CD163. Immunohistochemical staining revealed that the expression of CD163 ${ }^{+}$ macrophage is markedly elevated in the surrounding adipose tissue and the center of tumor tissue of breast cancer (Fig. 1A). Meanwhile, QDs-based fluorescent imaging showed that $C D 68^{+} C D 163^{+}$ macrophages remarkably infiltrated in adipocytes adjacent to tumor tissues and in connective tissue (Fig. 1B). In addition, CD11c is M1 and proinflammatory marker of ATMs[27], further D11 $\mathrm{c}^{+}$macrophages were also found to constitute the CLSs[28]. However, CD11c was extremely low expression and even no expression in our study (Fig. S1A). Our data demonstrated that the expression of CD163 in breast cancer specimens was associated with tumor size $(P=0.017)$, and it was greatly increased in patients with tumor size $\geq 2 \mathrm{~cm}$. In addition, the infiltration of $\mathrm{CD} 68^{+} \mathrm{CD} 163^{+}$macrophages were notably elevated when tumor metastasized into lymph node $(P<0.001)$. Moreover, the positive expression of CD163 was more common in patients overexpressed $\mathrm{Ki}-67$ compared to the control group $(P=0.007)$. There was no correlation between CD163 and other clinicopathological features, including age, vascular invasion, hormone receptors and human epithelial growth factor receptor-2 (HER2). 
We further analyzed the association between the expression of $\mathrm{CD} 68^{+} \mathrm{CD} 163^{+}$macrophages and prognosis of patients with breast cancer. We calculated the infiltration of $\mathrm{CD} 68^{+} \mathrm{CD} 163^{+}$macrophages in adipocytes adjacent to tumor tissues and in tumor tissues, respectively. The Kaplan-Meier survival analysis showed that the high expression of $\mathrm{CD} 68^{+} \mathrm{CD} 163^{+}$macrophages in adipocytes was significantly associated with shorter recurrence-free survival (RFS) than that of patients with negative expression ( $\mathrm{P}=$ 0.0142, Fig. 1C left). Whereas, there was no significance in RFS between CD163-negative and -positive patients grouping via the $\mathrm{CD} 163^{+}$macrophages infiltration in tumor tissues ( $P=0.316$, Fig. $1 \mathrm{C}$ right). These results demonstrated that macrophages surrounded adipocytes polarized towards M2-like phenotype and high level of infiltrated $\mathrm{CD} 68^{+} \mathrm{CD} 163^{+}$macrophages in adipose adjacent to tumor tissues was associated with various clinicopathologic parameters and poor prognosis in breast cancer patients. Taken together, these data indicate that the level of $\mathrm{CD}_{163^{+}}$macrophages were up-regulated in tumoradipose microenvironment and contributed to tumor progression.

\section{Chemokines CCL2 and CCL5 contribute to recruit macrophages.}

Our previous study and others have shown that the secreted levels of CCL2 and CCL5 elevated in adipocytes cocultured with tumor cells[22,29]. Driven the fact that macrophages could be recruited to inflammatory regions by chemokines, including CCL2[30] and CCL5[18], we supposed that macrophages surrounded by adipocytes were recruited by CCL2 and CCL5 to adipose tissue. Of 145 breast cancer specimens in our study, we found that the chemokines, both CCL2 and CCL5, overexpressed around the adipose tissues (Fig. 2A) and contributed to a poor survival (Fig. 2B). We next sought to determine whether macrophages might be an important target of CCL2 and CCL5 within breast tumors. Indeed, the proteins expression of both CCL2 and CCL5 are correlated with expression of CD68 + CD163 + macrophages (Fig. $2 C, R=0.211, P=0.011 ; R=0.261, P=0.001$, respectively), suggesting that $C L 2$ and CCL5 may attract macrophage migration during the formation of CLSs.

In vitro, after the presence of MDA-MB-231 cultivated for 3 days in Transwell system, the mRNA levels of CCL2 and CCL5 in adipocytes are dramatically elevated (Fig. 3A-B). Likewise, the conditioned media (CM) from mature adipocytes in the presence or absence of breast cancer cells (4T1, MDA-MB-231) cultivated for 3 days were collected. And it shows that CCL2 and CCL5 are highly expressed in CM from mature adipocytes co-cultivated with MDA-MB-231 cells via ELISA assay (Fig. 3C). Notably, the migration capabilities of macrophages also increased in co-cultivated group (Fig. 3D). To sum up, these results have indicated that CCL2 and CCL5 play a crucial role on macrophages recruit.

\section{Chemokines CCL2 and CCL5 induced M2-like polarization of macrophages via activating STAT3 phosphorylation}

Prominently, it has evidence that chemokines CCL2 and CCL5 enable the selective polarization of subsets of macrophages via interacting their chemokine receptors [16]. 
To determine how macrophages encapsulated and polarized in CLSs, we explore the impact of CCL2 and CCL5 on macrophages polarity. Next we confirmed that both mouse macrophages (RAW264.7 cell)[31] and human monocyte-derived macrophages (THP-1 cell induced by phorbol-12-myristate-13-acetate (PMA)[32]) showed increased M2-like (CD163) polarization upon treatment of CM from adipocytes cocultured with breast cancer cells relative to the single cultivation (Fig. 3E-F). In terms of mechanism, the expression of pSTAT3 was increased in macrophages with treatment of cocultured CM (Fig. 3G). To explore the impact of chemokines on macrophages polarization, the neutralizing antibodies targeted CCL2 and CCL 5 respectively were adopted. The results demonstrated that the polarized markers of macrophages and the expression of pSTAT3 were reversed relative to control group (Fig. 3H-I, Fig. S2A). In summary, our results demonstrated that the increase of CCL2 and CCL5 in cocultured CM promoted M2-like polarization of macrophages in vitro through phosphorylating STAT3.

\section{Tumor-derived exosomal miR-155 stimulates CCL2 and CCL5 secretion by adipocytes to accelerate tumour growth}

To confirm that the observed reliance for macrophages in CLSs depends on the tumor-adipocytes interaction, we examined whether the conditional presence of cancer cells alters the secreted spectrum of adipocytes. Based on our previous work, we speculated that the exosomes derived from tumour cells are important for adipocytes that are converted into cancer-associated adipocytes (CAAs) and excessively secrete chemokines including CCL5 [21-23]. Exosomes were isolated from conditioned medium, displaying that typical exosomal morphology and size and contained CD63, TSG101 and CD81 but not HSP70 (Fig. S3A-C), which was consistent with previous reports on exosomes [33]. To observe exosomes uptake by adipocytes, breast cancer-secreted exosomes were labeled with red fluorescence. After being treated with exosomes for 4 hours, mature 3T3-L1 cells were densely packed with exosomes (Fig. S3D), indicating rapid cellular uptake of exosomes by adipocytes. Further, exosomal miR-155 upregulated in cocultured group. Meanwhile, the mature miR-155 also overexpressed in adipocytes in presence of breast cancer cells, but the precursor of miR-155 remains unchanged (Fig. 4A). It suggested that exosomal miR155 , the suitable candidate, mainly released from breast cancer cells. What's more, the secretion levels of CCL2 and CCL 5 are strongly elevated in CM from mature adipocytes cocultivated with MDA-MB-231 cells, which are reversed by miR-155 knockdown (Fig. 4B).

This observed increase in exosomal miR-155 prompted us to analyze the effects of tumor-originated miR155 on the inflammation change of adipocytes. Intriguingly, we found one potential sequence of miR-155 targeting the human suppressor of cytokine signaling 6 (SOCS6) 3'UTR sequence (Fig. 4C). We next established one luciferase reporter, displaying that the wild-type construct showed a significant decrease in normalized luciferase activity relative to the control in the presence of pre-miR-155, and that mutation of the 3'UTR of human SOCS6 rescued this luciferase activity (Fig. 4D). This result shows that SOCS6 is a direct target of miR-155. We also detected the mRNA and protein expression of SOCS6, and it was evident that the generation of SOCS6 was decreased in adipocytes co-cultivated with breast cancer cells (Fig. 4E-F). SOCS6 is negative regulator of Janus activated kinase (JAK)/signal transducer and activator of transcription (STAT) signaling pathway[34], and the reduction of SOCS6 nuclear localization promoted 
protein expression of STAT3[35]. This was consistent with our results, showing that pSTAT3 level was extremely increased in mature adipocytes in the presence of breast cancer cells, which was reversed by cytochalasin D (CytoD), an endocytosis inhibitor (Fig. 4F-G). This result indicated that the exosome uptake contributes to the upregulation of pSTAT3 in adipocytes undergoing tumour cells. Further, we investigated the possibility that exo-miR-155 would activate PSTAT3 activity via inhibiting SOCS6. The results indicated that the inhibition of SOCS6 activity in adipocytes cocultivated with breast cancer cells was comparable to that of miR-155 overexpression (Fig. 4H-I). This inhibitory effect was achieved through miR-155 knockdown (Fig. 4H-I). Consistently, the administration in co-cultured adipocytes increased the SOCS6-mediated phosphorylation of STAT3 and the knock-downed miR-155 would reverse the expression (Fig. $4 \mathrm{H}-\mathrm{I})$. The xenograft models showed consistent results with in vitro assays, indicating that significantly increased tumour growth in mice co-injected with 4T1 cells and mature 3T3-L1 cells, whereas downregulation of miR-155 in tumour-derived exosomes significantly reduced the tumour volume in xenografts (Fig. 4J-L). Likewise, the number and the size of metastatic nodules in liver were markedly increased in the group injected with tumour cells cocultivated with mature 3T3-L1 cells, while downregulation of miR-155 in tumour exosomes significantly reduced the number of nodules in liver (Fig. 4L). And the number and the size of metastatic nodules in lung were no obvious difference among these groups (data not shown). Altogether, our results demonstrated that exosomal miR-155 derived from breast cancer cells mediated inflammatory adaptive changes in mature adipocytes through SOCS6/STAT3 signaling pathway.

\section{Macrophages deficiency in tumor-adipocyte interaction inhibits tumor growth}

Whether macrophages existence endowed breast cancer cells with the proliferated capacity, we speculated that macrophages deficiency might inhibit adipocyte-associated tumor growth. To this end, we employed mouse model without macrophages to assess the impact of deficient macrophages on breast cancer proliferation. On account that macrophages in adipose and tumor tissues are mixed by tissue-resident macrophages and monocyte-derived cells, anti-F4/80 mAb treatment could delete multifarious macrophages and be used in further study. As our previous studies, 3T3-L1 cells were induced to differentiate into mature adipocytes. Furthermore, a kind of breast cancer cell from BALB/C mouse $4 \mathrm{~T} 1$ with or without mature adipocytes in a specific proportion were orthotopically injected into the mammary fat pad[22]. Likewise, the macrophages in mouse model were particularly eliminated via injection of anti-F4/80 neutralizing antibody as reported[13] (Fig. 5A). As Fig. 5B-C, the tumor injected 4T1 combined with adipocytes grew much rapidly while the eliminated macrophages via anti-F4/80 mAb could significantly reduce these tumors growth. To determine whether M2-like phenotype of macrophages represents a general mechanism for promoting tumor progression, we further evaluated the number and biomarkers of macrophages in this model. From IHC stained images shown in Fig. 5D, the infiltration of $\mathrm{CD}_{163^{+}}$macrophages were remarkably elevated in mice injected 4T1 with adipocytes, by contrast, antiF4/80 mAb dramatically alleviated the macrophages recruitment. Consistently, pathological grade in samples of the co-injected group was much higher than those in groups receiving anti-F4/80 mAb 
treatment. These data collectively indicated that macrophages deficiency in adipose tissues inhibited adipocyte-promoted tumor growth.

\section{Blocking the chemokines/receptors/STAT3 axis prevents breast cancer growth}

To investigate the role of CCL2-CCR2 and CCL5-CCR5 axis and their downstream key regulator STAT3 on tumor progression in vivo, we inoculated the mouse mammary carcinoma cells AT3 in the presence or absence of mature adipocytes into the mammary fat pads of C57BL/ 6 mice ( $n=6$ per group), and evaluated tumor growth. When the xenografts became palpable, CCL2- or CCL5-specific neutralizing antibodies as described in Fig. 6A were injected via the tail vein. BMS-813160, the CCR2/CCR5 inhibitor[36], and Stattic, STAT3 inhibitor[37], were also used to estimate the potential therapeutic effect. It found that compared with AT3 cells alone, combined injection of mature 3T3-L1 accelerated the tumor growth of xenografts $(\mathrm{P}<0.05$; Fig. 6B). CCL2- or CCL5-specific neutralizing antibodies, as well as BMS813160 and Stattic, markedly retarded the tumor proliferation of xenografts in the malignant AT3 cells plus mature adipocytes inoculation groups $(P<0.05$, Fig. 6B-E). Meanwhile, the decreasing infiltration of $\mathrm{CD}_{163^{+}}$macrophages were discovered among these groups treated as described above (Fig. 6F).

Exosome originated from tumor-adipocyte interplay induce autophagy in macrophages depending on transcription factor 3 (TFE3) translocation

Recent study demonstrates that high expression of CD163 is a prominent biomarker of M2c phenotype of macrophages, thus giving rise to their efficient phagocytosis of apoptotic cells[38]. Meanwhile, activated autophagy conduces to enhance phagocytosis of macrophages[39]. Using RAW264.7 cells stably expressing GFP-LC3, we found that the isolated exosomes enhances the level of LC3 II and GFP-LC3 dots, then the expression of GFP-LC3 dots and the lipidation of LC3 was further increased in the presence of the lysosomal inhibitor Bafilomycin A1(Baf-A1) (Fig. S5A-C). Likewise, the exosomes caused a reduction in the abundance of P62, and this effect was blocked by Baf-A1 (Fig. S5B). In addition, the result showed that the extractive exosomes enabled to stimulate the translocation of TFE3 from the cytoplasm to the nucleus (Fig. S5D-F). Further, knockdown of TFE3 with siRNA (Fig. S5G) partially reduced the formation of GFP-LC3 puncta in response to exosomes (Fig. S5H). Similarly, The TFE3 KD was particularly efficient in reducing exosomes-induced LC3 lipidation (Fig. S5I-J). In sum, the exosomes from tumor-adipocyte coculture stimulated autophagy by the activation of TFE3.

\section{Discussion}

Macrophages are particularly important in adipose tissue, and contribute to link obesity-related inflammation and tumor progression. Likewise, macrophages envelope around phagocytosis of a dead or dying adipocyte, this configuration is termed as crown-like structures (CLS) [40]. Notably, the number and the density of CLS is positively correlated to high body mass index, large adipocyte size, postmenopausal status as well as insulin resistance in obese subjects, suggesting the pathophysiologic role CLS played in 
adipose tissue inflammation[26, 41]. Hence, macrophages infiltration in adipose tissue around tumor seems to have vital clinical significance, especially in macrophage polarization, yet it needs further investigation. The present study describes that exosomal miRNA-155 from the tumor-adipocyte crosstalk increase the secretion of CCL2 and CCL5 in adipocytes, further the chemokines recruit macrophages around the adipocytes and repolarized macrophages towards M2-like subtype. Not surprisingly, deleting macrophages in this context retard tumor growth.

Macrophages are a complicated kind of cells, which express diverse surface markers and have unique anatomical locations[7, 42]. Macrophages are demonstrated to develop from three distinct origins including embryonic precursors, adult haematopoietic stem cells (HSCs) or bone marrow derived monocytes [43]. Likewise, two main phenotypes of macrophages are the classical polarized M1 macrophages and alternatively polarized M2 macrophages[9]. CD11c is discovered as pre- inflammatory biomarkers especially expressed in tissue-resident macrophages[44] while M2 macrophages have an antiinflammatory effect via up-regulating CD163, CD206 and CD204 [11, 42]. Meanwhile, it frequently exists macrophages reprogramming from one subset into another, partly explaining this diversity of macrophages[45]. In addition, macrophage phenotype can vary from different cancer types and intratumor districts[11]. Our results have shown that $C D 163^{+}$macrophages not CD11 ${ }^{+}$ATMs highly infiltrating in tumor-adipose regions predicted poor survival in patients undergoing breast cancer. It suggests that the macrophages mainly derived from monocyte-derived cells not tissue-resident macrophages. Importantly, M2 macrophages could be activated into an M2c phenotype, which high expression of CD163 and Mer receptor tyrosine kinase (MerTK), thus giving rise to their efficient phagocytosis of apoptotic cells[46]. Described above, macrophages wrap and engulf the dead or dying adipocytes in CLS, suggesting that M2c phenotype might be potential subset of macrophage in this condition. However, M2a macrophages, another subtype of M2 macrophages, express high levels of CD206 but not CD163. These M2a macrophages enrich genes associated with tissue remodeling and wound healing[47]. Taken together, CD163, one of special surface markers expressed M2c macrophage, may be the optimal biomarker in CLS. Importantly, tissue-resident M2c macrophages in tumor tissues enrich genes associated with tissue remodeling, and have increased expression of genes concerning immunosuppression[38]. By contrast, M2a macrophages mainly exert anti-inflammatory and woundhealing effects[47] while M2b macrophages, functioned as immunoregulation, secrete pro- and antiinflammatory cytokines meantime and are found in tumors[38, 48, 49]. Finally, M2d macrophages represent a novel M2 subset and constitute the major inflammatory component in neoplastic tissue, contributing to angiogenesis and cancer metastasis[50]. However, adipose tissues and cancer contain macrophages with a mixed subtypes[8,38]. Thus, comprehending the heterogeneity and plasticity of macrophage phenotypes is pivotal for obesity-related cancer.

The chemokines such as CCL2 and CCL5 have the pivotal functions in the recruit and polarization of macrophages in both tumor tissues and fatty tissues[7, 10, 41]. In the case of obesity, the hypertrophic expansion of adipose tissue has many features in common with the growth of solid tumors. The hypoxia in obese adipose tissue induces expression of the transcription factor HIF-1a, which is consistent with 
tumor hypoxia, thereby increasing expression of proinflammatory cytokines including CCL2 and CCL5[51, 52]. The present results indicate that CLL2 and CCL5 overexpress around the adipose tissues and are positive correlated with $\mathrm{CD}_{163^{+}}$macrophages infiltration in breast cancer specimens, further promoting M2-like polarization of macrophages via activating pSTAT3. Hypertrophic adipocyte-derived chemotactic CCL2/CC chemokine receptor 2 (CCR2) pathway recruits more macrophages to accumulate in the obese adipose tissue. Subsequently, CCL2 polarizes macrophages toward the M2 phenotype [53]. Additionally, CCL2 enhances the LPS-induced expression of IL-10 in macrophages, while CCL2 blockade results in increased generation of M1 polarization-related genes and cytokines, and decreased generation of M2related markers in human macrophages[54]. Likewise, CCL5 is elevated and remains high in adipose microenvironment[55], and promotes tumor recurrence by recruiting CCR5-expressing macrophages, which may contribute to collagen deposition in residual tumors[18]. Moreover, CCL5 activates AKT signaling to recruit and repolarize TAMs via bounding to its receptor, CCR5[56]. The CCR5 inhibitors prevent recruitment of monocytes to the tumor and repolarize macrophages with anti-tumoral effects [56, 57]. Therefore, CCL2 and CCL5 not only recruit macrophages to the tumor or obesity-induced microenvironment but shape M2-skewed polarization of macrophages.

Exosome has been defined as a novel way for cell-to-cell communication that interact with a neighbor or distant target cells[19]. Potentially, the exosome-contained specific contents like miRNAs as potential biomarkers and the amount of released exosomes are altered in obesity and cancer. In current results, exosomal miRNA-155 suppresses SOCS6, a negative regulator of the JAK-STAT signaling pathway, further induces the phosphorylation of STAT3 that contributes to the secretion of CCL2 and CCL5. Inhibition of miRNA-155 suppresses the generation of CCL2 and CCL5 and blocks adipocytes-induced tumor growth. Our previous studies have demonstrated that exosomes from the tumor-adipocyte symbiosis contain several specific miRNAs such as miRNA-126, miRNA-144 and miRNA-155[21-23]. Indeed, these exosomal miRNAs play pivotal roles in mediating the differentiation and functions of macrophages[58-61]. For example, the up-regulation of miRNA-155 in macrophages increases CCL2 secretion through directly inhibiting B-cell lymphoma-6 (BCL6) expression and the decreased expression of Arginase- 1 and Chil3[58, 62]. Additionally, cancer cell-secreted miRNA-155 targets PPARY and downregulates its level[22, 23]. Meanwhile, a PPARy ligand rosiglitazone converts high fat-induced M2 polarization of macrophages toward anti-inflammatory subtype[63]. By contrast, propranolol, a nonselective sympatholytic $\beta$-blocker, not only alleviates tumor exosome-stimulated cachexia via activating PPARy[23] but also inhibits the production of IL-10 in M2 macrophages[64]. The specific effects of PPAR $y$ activators in inhibiting M2 macrophages polarization and the efficacy of these compounds in immunologic/inflammatory diseases requires to further be demonstrated. Taken together, exosomal miRNAs originated from tumor cells may adequately reshape adipocytes in fatty microenvironment to convert macrophages into a pro-tumor niche.

Autophagy has multiple effects on macrophages. On the one hand, autophagy activity in macrophages enhance IL-1 $\beta$ secretion[65]. On the other hand, autophagy may render macrophage phagocytosis[66]. The basic helix-loop-helix transcription factor TFE3 (transcription factor 3) belongs to the MiT/TFE 
family[67]. It has evidence that TFE3 controls lysosomal biogenesis and autophagy by regulating the expression of several lysosomal and autophagic genes[68]. Normally, TFE3 is located in the cytoplasm and translocates to the nucleus in response to various stress[67]. Importantly, TFE3 is crucial for macrophages activation via inducing autophagy, increasing lysosomal biogenesis, and upregulating numerous proinflammatory cytokines in transcriptional way including CCL2 and CCL5[69]. Transcription factor EB (TFEB) is similar with TFE3 $[67,69]$, and its expression elevates in IL-4 induced M2 macrophages, but reduces in tumor-conditioned medium-treated macrophages. Likewise, TFEB ablation promotes breast tumor growth[70]. Interesting, recent study shows that TFEB reduction was accompanied in some instances by a concomitant increase in TFE3 levels, which might be indicative of a compensatory mechanism between both transcription factors[69]. Consistent with our results, TFE3 but not TFEB promote autophagic flux in activated macrophages.

\section{Conclusion}

In summary, as depicted in model (Fig. 7), we put forward a potential mechanism that exosomal miR-155 from tumor-adipocyte interplay could promote the secretion of CCL2 and CCL5 in adjacent adipocytes via targeting SOCS6/STAT3 signaling, subsequently accumulating M2-like subtype of macrophages towards tumor-adipose microenvironment. Together, these studies shed light on targeting strategies for reducing interaction among tumors, stromal cells and immune cells as a method to clarified adipocytemacrophage-tumor interactions in planning treatment strategies of obesity-associated cancer.

\section{Declarations}

\section{Ethics approval and consent to participate}

All patients involved in the study provided a written informed consent, and the study was approved by the Institutional Ethics Committee of Renmin Hospital of Wuhan University (approval no. 2018K-C09).

\section{Consent for publication}

Not applicable

\section{Availability of data and materials}

The datasets used and analyzed during the current study are available from the corresponding author on reasonable request.

\section{Competing interests}

The authors declare that they have no conflicts of interest.

\section{Funding}


This work was supported by a National Natural Science Foundation of China (NSFC) grant (Grant NO: 81471781) to Prof. Shengrong Sun and (Grant NO: 81903166) to Dr. Si Sun. This work was also supported by a Hubei Province health and family planning scientific research project to Dr. Si Sun (Grant No. WJ2019Q044), and a project (Grant No. WJ2019M188) to Dr. Zhiliang Xu. Finally, this work was supported by the Natural Science Foundation of Hubei (grant No. 2018CKB916) to Dr. Si Sun.

\section{Authors' contributions}

Q. Wu, B. Li, and Q. Yang are responsible for conception and design. Q. Wu, B. Li, ZY. Li, and J. Wu are responsible for the development of methodology. JJ. Li, S. Zhu, LJ. Wang, SC. Liao, ZL. Xu, and S. Sun are responsible for the acquisition of data. B. Li and Q. Wu are responsible for the analysis and interpretation of data. B. Li, Q. Wu and SR. Sun are responsible for writing, review, and/or revision of the manuscript. Acknowledgments

The authors thank all the patients and the patients' families for their corporation. We thank a professional English editor (American Journal Experts) for assistance in improving the quality of language.

\section{Abbreviations}

AD-CM: adipocyte-conditioned medium; BCL6: B-cell lymphoma-6; BMI: body mass index; ATM: adipose tissue macrophages; CAAs: cancer-associated adipocytes; CA-CM: cancer-associated adipocyte conditioned medium; CCL: C-C chemokine ligand; CCR: $\mathrm{C}-\mathrm{C}$ chemokine receptor; Chil3: chitinase-like protein 3; CLSs: crown-like structures; CM: conditioned media; CytoD: cytochalasin D; HER2: human epidermal growth factor receptor-2; HE: haematoxylin-eosin; IF: Immunofluorescence; IHC: Immunohistochemistry; JAK: Janus activated kinase; MerTK: Mer receptor tyrosine kinase; PPARy: peroxisome proliferator-activated receptor Y; RFS: recurrence-free survival; SOCS6: suppressor of cytokine signaling 6; STAT: signal transducer and activator of transcription; TAMs: tumor-associated macrophages; TFE3: transcription factor 3; TFEB: Transcription factor EB.

\section{References}

1. Calle EE, Rodriguez C, Walker-Thurmond K, Thun MJ. Overweight, obesity, and mortality from cancer in a prospectively studied cohort of U.S. adults. N Engl J Med. 2003;348(17):1625-38.

2. Picon-Ruiz M, Morata-Tarifa C, Valle-Goffin JJ, Friedman ER, Slingerland JM. Obesity and adverse breast cancer risk and outcome: Mechanistic insights and strategies for intervention. Cancer $\mathrm{J}$ Clin. 2017;67(5):378-97.

3. Park J, Morley TS, Kim M, Clegg DJ, Scherer PE. Obesity and cancer-mechanisms underlying tumour progression and recurrence. Nature reviews Endocrinology. 2014;10(8):455-65.

4. lyengar NM, Gucalp A, Dannenberg AJ, Hudis CA. Obesity and Cancer Mechanisms: Tumor Microenvironment and Inflammation. Journal of clinical oncology: official journal of the American Society of Clinical Oncology. 2016;34(35):4270-6. 
5. Wu Q, Li B, Li Z, Li J, Sun S, Sun S. Cancer-associated adipocytes: key players in breast cancer progression. J Hematol Oncol. 2019;12(1):95.

6. Wu Q, Li B, Sun S, Sun S. Unraveling Adipocytes and Cancer Links: Is There a Role for Senescence? Frontiers in cell developmental biology. 2020;8:282.

7. Cox N, Geissmann F. Macrophage ontogeny in the control of adipose tissue biology. Curr Opin Immunol. 2019;62:1-8.

8. Wynn TA, Chawla A, Pollard JW. Macrophage biology in development, homeostasis and disease. Nature. 2013;496(7446):445-55.

9. Chylikova J, Dvorackova J, Tauber Z, Kamarad V. M1/M2 macrophage polarization in human obese adipose tissue. Biomedical papers of the Medical Faculty of the University Palacky Olomouc Czechoslovakia. 2018;162(2):79-82.

10. Deng T, Lyon CJ, Bergin S, Caligiuri MA, Hsueh WA. Obesity, Inflammation, and Cancer. Annual review of pathology. 2016;11:421-49.

11. Biswas SK, Mantovani A. Macrophage plasticity and interaction with lymphocyte subsets: cancer as a paradigm. Nat Immunol. 2010;11(10):889-96.

12. Shaul ME, Bennett G, Strissel KJ, Greenberg AS, Obin MS. Dynamic, M2-like remodeling phenotypes of $\mathrm{CD} 11 \mathrm{c}+$ adipose tissue macrophages during high-fat diet-induced obesity in mice. Diabetes. 2010;59(5):1171-81.

13. Jackaman C, Yeoh TL, Acuil ML, Gardner JK, Nelson DJ. Murine mesothelioma induces locallyproliferating IL-10(+)TNF-alpha(+)CD206(-)CX3CR1(+) M3 macrophages that can be selectively depleted by chemotherapy or immunotherapy. Oncolmmunology. 2016;5(6):e1173299.

14. Mullooly M, Yang HP, Falk RT, Nyante SJ, Cora R, Pfeiffer RM, Radisky DC, Visscher DW, Hartmann LC, Carter JM, et al. Relationship between crown-like structures and sex-steroid hormones in breast adipose tissue and serum among postmenopausal breast cancer patients. Breast cancer research: BCR. 2017;19(1):8.

15. Cha YJ, Kim ES, Koo JS. Tumor-associated macrophages and crown-like structures in adipose tissue in breast cancer. Breast Cancer Res Treat. 2018;170(1):15-25.

16. Ruytinx P, Proost P, Van Damme J, Struyf S. Chemokine-Induced Macrophage Polarization in Inflammatory Conditions. Frontiers in immunology. 2018;9:1930.

17. Brummer G, Fang W, Smart C, Zinda B, Alissa N, Berkland C, Miller D, Cheng N. CCR2 signaling in breast carcinoma cells promotes tumor growth and invasion by promoting CCL2 and suppressing CD154 effects on the angiogenic and immune microenvironments. Oncogene. 2020;39(11):2275-89.

18. Walens A, DiMarco AV, Lupo R, Kroger BR, Damrauer JS, Alvarez JV. CCL5 promotes breast cancer recurrence through macrophage recruitment in residual tumors. eL ife 2019, 8.

19. Tkach M, Thery C. Communication by Extracellular Vesicles: Where We Are and Where We Need to Go. Cell. 2016;164(6):1226-32. 
20. Latifkar A, Hur YH, Sanchez JC, Cerione RA, Antonyak MA. New insights into extracellular vesicle biogenesis and function. Journal of cell science 2019, 132(13).

21. Wu Q, Sun S, Li Z, Yang Q, Li B, Zhu S, Wang L, Wu J, Yuan J, Yang C, et al. Tumour-originated exosomal miR-155 triggers cancer-associated cachexia to promote tumour progression. Mol Cancer. 2018;17(1):155.

22. Wu Q, Li J, Li Z, Sun S, Zhu S, Wang L, Wu J, Yuan J, Zhang Y, Sun S, et al. Exosomes from the tumour-adipocyte interplay stimulate beige/brown differentiation and reprogram metabolism in stromal adipocytes to promote tumour progression. Journal of experimental clinical cancer research: CR. 2019;38(1):223.

23. Wu Q, Sun S, Li Z, Yang Q, Li B, Zhu S, Wang L, Wu J, Yuan J, Wang C, et al. Breast cancer-released exosomes trigger cancer-associated cachexia to promote tumor progression. Adipocyte. 2019;8(1):31-45.

24. Rider MA, Hurwitz SN, Meckes DG Jr. ExtraPEG: A Polyethylene Glycol-Based Method for Enrichment of Extracellular Vesicles. Scientific reports. 2016;6:23978.

25. Koru-Sengul T, Santander AM, Miao F, Sanchez LG, Jorda M, Gluck S, Ince TA, Nadji M, Chen Z, Penichet $\mathrm{ML}$, et al. Breast cancers from black women exhibit higher numbers of immunosuppressive macrophages with proliferative activity and of crown-like structures associated with lower survival compared to non-black Latinas and Caucasians. Breast cancer research treatment. 2016;158(1):113-26.

26. Cha YJ, Kim ES, Koo JS: Tumor-associated macrophages and crown-like structures in adipose tissue in breast cancer. Breast cancer research and treatment 2018.

27. Gambaro SE, Zubiria MG, Portales AE, Rey MA, Rumbo M, Giovambattista A. M1 macrophage subtypes activation and adipocyte dysfunction worsen during prolonged consumption of a fructoserich diet. J Nutr Biochem. 2018;61:173-82.

28. Itoh M, Suganami T, Kato H, Kanai S, Shirakawa I, Sakai T, Goto T, Asakawa M, Hidaka I, Sakugawa $H$, et al: CD11c + resident macrophages drive hepatocyte death-triggered liver fibrosis in a murine model of nonalcoholic steatohepatitis. JCl insight 2017, 2(22).

29. Dirat B, Bochet L, Dabek M, Daviaud D, Dauvillier S, Majed B, Wang YY, Meulle A, Salles B, Le Gonidec $S$, et al. Cancer-associated adipocytes exhibit an activated phenotype and contribute to breast cancer invasion. Cancer research. 2011;71(7):2455-65.

30. Sun X, Glynn DJ, Hodson LJ, Huo C, Britt K, Thompson EW, Woolford L, Evdokiou A, Pollard JW, Robertson SA, et al. CCL2-driven inflammation increases mammary gland stromal density and cancer susceptibility in a transgenic mouse model. Breast Cancer Res. 2017;19(1):4.

31. Raschke WC, Baird S, Ralph P, Nakoinz I. Functional macrophage cell lines transformed by Abelson leukemia virus. Cell. 1978;15(1):261-7.

32. Auwerx J. The human leukemia cell line, THP-1: a multifacetted model for the study of monocytemacrophage differentiation. Experientia. 1991;47(1):22-31. 
33. Mashouri L, Yousefi H, Aref AR, Ahadi AM, Molaei F, Alahari SK. Exosomes: composition, biogenesis, and mechanisms in cancer metastasis and drug resistance. Mol Cancer. 2019;18(1):75.

34. Shen R, Wang Y, Wang CX, Yin M, Liu HL, Chen JP, Han JQ, Wang WB. MiRNA-155 mediates TAM resistance by modulating SOCS6-STAT3 signalling pathway in breast cancer. Am J Transl Res. 2015;7(10):2115-26.

35. Hwang MN, Min CH, Kim HS, Lee H, Yoon KA, Park SY, Lee ES, Yoon S. The nuclear localization of SOCS6 requires the $\mathrm{N}$-terminal region and negatively regulates Stat3 protein levels. Biochem Biophys Res Commun. 2007;360(2):333-8.

36. Norman P. A dual CCR2/CCR5 chemokine antagonist, BMS-813160? Evaluation of W02011046916. Expert Opin Ther Pat. 2011;21(12):1919-24.

37. Yang H, Yamazaki T, Pietrocola F, Zhou H, Zitvogel L, Ma Y, Kroemer G. STAT3 Inhibition Enhances the Therapeutic Efficacy of Immunogenic Chemotherapy by Stimulating Type 1 Interferon Production by Cancer Cells. Cancer research. 2015;75(18):3812-22.

38. Wang LX, Zhang SX, Wu HJ, Rong XL, Guo J. M2b macrophage polarization and its roles in diseases. J Leukoc Biol. 2019;106(2):345-58.

39. Ma Y, Galluzzi L, Zitvogel L, Kroemer G. Autophagy and cellular immune responses. Immunity. 2013;39(2):211-27.

40. Howe LR, Subbaramaiah K, Hudis CA, Dannenberg AJ. Molecular pathways: adipose inflammation as a mediator of obesity-associated cancer. Clinical cancer research: an official journal of the American Association for Cancer Research. 2013;19(22):6074-83.

41. Engin AB, Engin A, Gonul II. The effect of adipocyte-macrophage crosstalk in obesity-related breast cancer. J Mol Endocrinol. 2019;62(3):R201-22.

42. DeNardo DG, Ruffell B. Macrophages as regulators of tumour immunity and immunotherapy. Nature reviews Immunology. 2019;19(6):369-82.

43. DeNardo DG, Ruffell B. Macrophages as regulators of tumour immunity and immunotherapy. Nature reviews Immunology 2019.

44. Morris DL, Singer K, Lumeng CN. Adipose tissue macrophages: phenotypic plasticity and diversity in lean and obese states. Curr Opin Clin Nutr Metab Care. 2011;14(4):341-6.

45. Perdiguero EG, Geissmann F. The development and maintenance of resident macrophages. Nat Immunol. 2016;17(1):2-8.

46. Zizzo G, Hilliard BA, Monestier M, Cohen PL. Efficient clearance of early apoptotic cells by human macrophages requires M2c polarization and MerTK induction. Journal of immunology. 2012;189(7):3508-20.

47. Wynn TA, Vannella KM. Macrophages in Tissue Repair, Regeneration, and Fibrosis. Immunity. 2016;44(3):450-62.

48. Mosser DM, Edwards JP. Exploring the full spectrum of macrophage activation. Nature reviews Immunology. 2008;8(12):958-69. 
49. Anderson CF, Mosser DM. A novel phenotype for an activated macrophage: the type 2 activated macrophage. J Leukoc Biol. 2002;72(1):101-6.

50. Wang Q, Ni H, Lan L, Wei X, Xiang R, Wang Y. Fra-1 protooncogene regulates IL-6 expression in macrophages and promotes the generation of M2d macrophages. Cell research. 2010;20(6):701-12.

51. Sun K, Tordjman J, Clement K, Scherer PE. Fibrosis and adipose tissue dysfunction. Cell Metabol. 2013;18(4):470-7.

52. Koh MY, Gagea M, Sargis T, Lemos R Jr, Grandjean G, Charbono A, Bekiaris V, Sedy J, Kiriakova G, Liu $X$, et al. A new HIF-1alpha/RANTES-driven pathway to hepatocellular carcinoma mediated by germline haploinsufficiency of SART1/HAF in mice. Hepatology. 2016;63(5):1576-91.

53. Roca H, Varsos ZS, Sud S, Craig MJ, Ying C, Pienta KJ. CCL2 and interleukin-6 promote survival of human CD11b + peripheral blood mononuclear cells and induce M2-type macrophage polarization. $J$ Biol Chem. 2009;284(49):34342-54.

54. Sierra-Filardi E, Nieto C, Dominguez-Soto A, Barroso R, Sanchez-Mateos P, Puig-Kroger A, Lopez-Bravo $M$, Joven J, Ardavin C, Rodriguez-Fernandez JL, et al. CCL2 shapes macrophage polarization by GMCSF and M-CSF: identification of CCL2/CCR2-dependent gene expression profile. Journal of immunology. 2014;192(8):3858-67.

55. D'Esposito V, Liguoro D, Ambrosio MR, Collina F, Cantile M, Spinelli R, Raciti GA, Miele C, Valentino R, Campiglia $\mathrm{P}$, et al. Adipose microenvironment promotes triple negative breast cancer cell invasiveness and dissemination by producing CCL5. Oncotarget. 2016;7(17):24495-509.

56. Nie Y, Huang H, Guo M, Chen J, Wu W, Li W, Xu X, Lin X, Fu W, Yao Y, et al. Breast Phyllodes Tumors Recruit and Repolarize Tumor-Associated Macrophages via Secreting CCL5 to Promote Malignant Progression, Which Can Be Inhibited by CCR5 Inhibition Therapy. Clinical cancer research: an official journal of the American Association for Cancer Research. 2019;25(13):3873-86.

57. Halama N, Zoernig I, Berthel A, Kahlert C, Klupp F, Suarez-Carmona M, Suetterlin T, Brand K, Krauss J, Lasitschka F, et al. Tumoral Immune Cell Exploitation in Colorectal Cancer Metastases Can Be Targeted Effectively by Anti-CCR5 Therapy in Cancer Patients. Cancer cell. 2016;29(4):587-601.

58. Nie M, Liu J, Yang Q, Seok HY, Hu X, Deng ZL, Wang DZ. MicroRNA-155 facilitates skeletal muscle regeneration by balancing pro- and anti-inflammatory macrophages. Cell death disease. 2016;7(6):e2261.

59. Liu HY. Down-regulation of miR-144 after Mycobacterium tuberculosis infection promotes inflammatory factor secretion from macrophages through the Tpl2/ERK pathway. Cell Mol Biol (Noisy-le-grand). 2016;62(2):87-93.

60. Zhang Y, Yang P, Sun T, Li D, Xu X, Rui Y, Li C, Chong M, Ibrahim T, Mercatali L, et al. miR-126 and miR$126^{*}$ repress recruitment of mesenchymal stem cells and inflammatory monocytes to inhibit breast cancer metastasis. Nat Cell Biol. 2013;15(3):284-94.

61. Zhou G, Li Y, Ni J, Jiang P, Bao Z. Role and mechanism of miR-144-5p in LPS-induced macrophages. Experimental therapeutic medicine. 2020;19(1):241-7. 
62. Nazari-Jahantigh M, Wei Y, Noels H, Akhtar S, Zhou Z, Koenen RR, Heyll K, Gremse F, Kiessling F, Grommes $\mathrm{J}$, et al. MicroRNA-155 promotes atherosclerosis by repressing Bcl6 in macrophages. J Clin Investig. 2012;122(11):4190-202.

63. Lefevre L, Gales A, Olagnier D, Bernad J, Perez L, Burcelin R, Valentin A, Auwerx J, Pipy B, Coste A. PPARgamma ligands switched high fat diet-induced macrophage M2b polarization toward M2a thereby improving intestinal Candida elimination. PloS one. 2010;5(9):e12828.

64. Kobayashi M, Jeschke MG, Asai A, Kogiso M, Yoshida S, Herndon DN, Suzuki F. Propranolol as a modulator of M2b monocytes in severely burned patients. J Leukoc Biol. 2011;89(5):797-803.

65. Dupont N, Jiang S, Pilli M, Ornatowski W, Bhattacharya D, Deretic V. Autophagy-based unconventional secretory pathway for extracellular delivery of IL-1 beta. Embo J. 2011;30(23):470111.

66. Germic N, Frangez Z, Yousefi S, Simon HU. Regulation of the innate immune system by autophagy: monocytes, macrophages, dendritic cells and antigen presentation. Cell Death Differ. 2019;26(4):715-27.

67. Raben N, Puertollano R. TFEB and TFE3: Linking Lysosomes to Cellular Adaptation to Stress. Annu Rev Cell Dev Biol. 2016;32:255-78.

68. Chen G, Xie W, Nah J, Sauvat A, Liu P, Pietrocola F, Sica V, Carmona-Gutierrez D, Zimmermann A, Pendl T, et al. 3,4-Dimethoxychalcone induces autophagy through activation of the transcription factors TFE3 and TFEB. EMBO Mol Med. 2019;11(11):e10469.

69. Pastore N, Brady OA, Diab HI, Martina JA, Sun L, Huynh T, Lim JA, Zare H, Raben N, Ballabio A, et al. TFEB and TFE3 cooperate in the regulation of the innate immune response in activated macrophages. Autophagy. 2016;12(8):1240-58.

70. Li Y, Hodge J, Liu Q, Wang J, Wang Y, Evans TD, Altomare D, Yao Y, Murphy EA, Razani B, et al: TFEB is a master regulator of tumor-associated macrophages in breast cancer. Journal for immunotherapy of cancer 2020, 8(1).

\section{Figures}


A
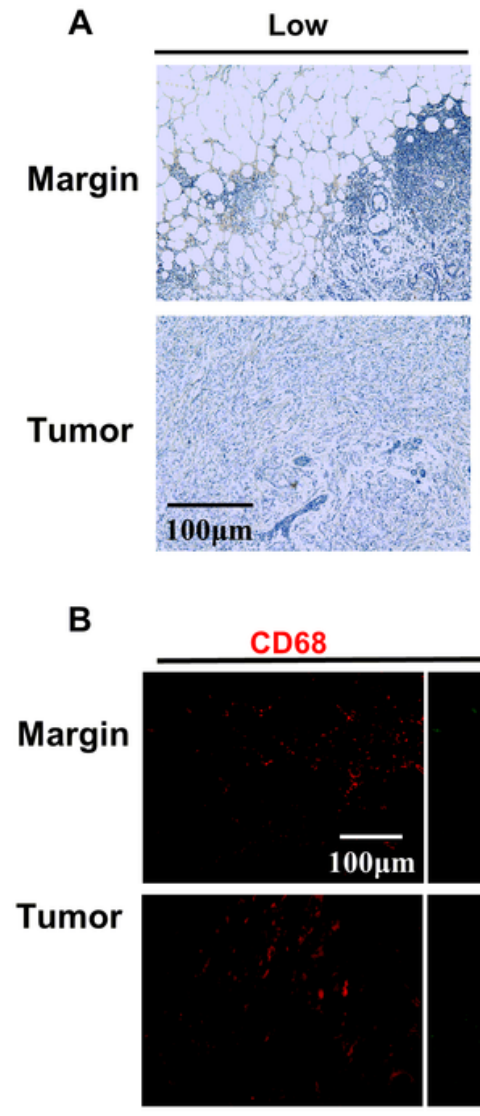

High

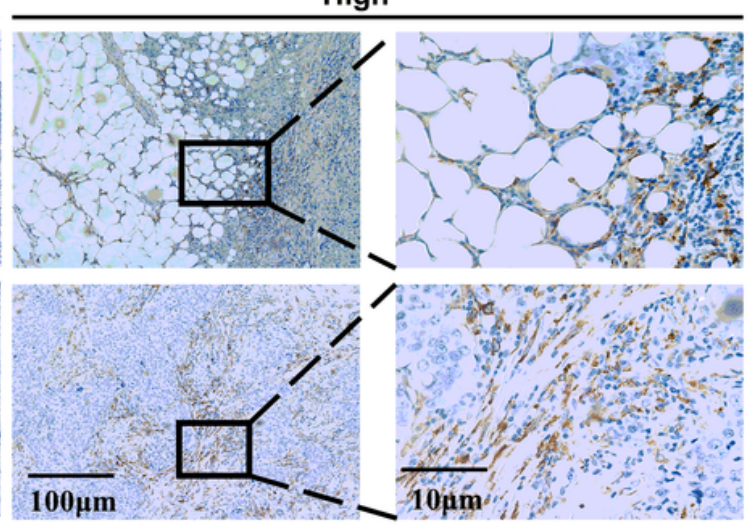

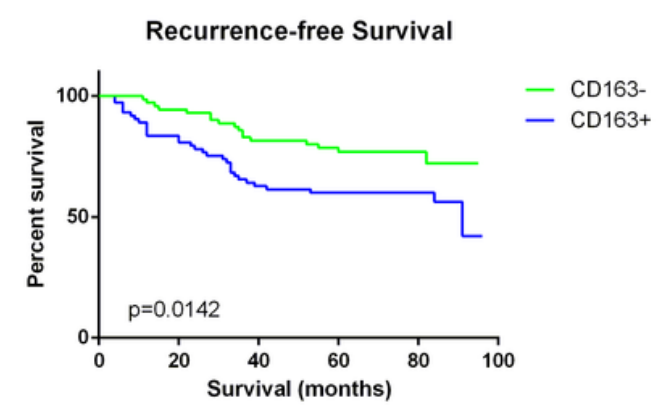

Margin

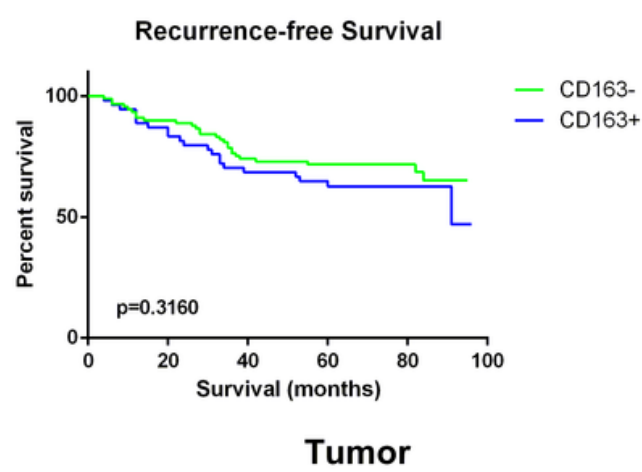

\section{Figure 1}

High infiltration of CD163+ macrophages in adipose tissues was correlated to poor prognosis in patients with breast cancer. A. Representative immunohistochemistry staining of CD163+ macrophages, the positive staining of which was located both in the breast cancer malignant tissues and the invasive margin near adipocytes. B. Immunofluorescence staining for CD68+CD163+ macrophages around the adipocytes adjacent to tumor tissues and in connective tissues. C. Kaplan-Meier survival analysis of patients with CD163-positive and -negative IHC staining in the margin and in the tissue, respectively. 


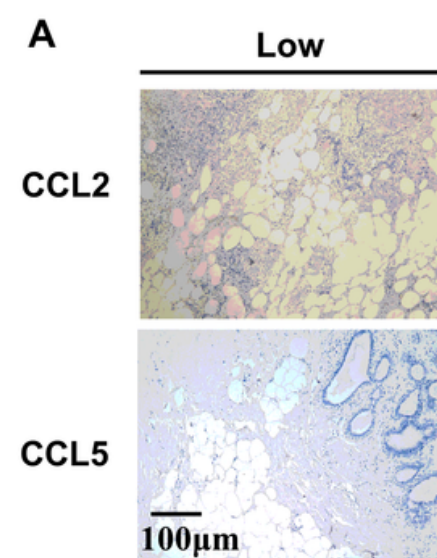

B

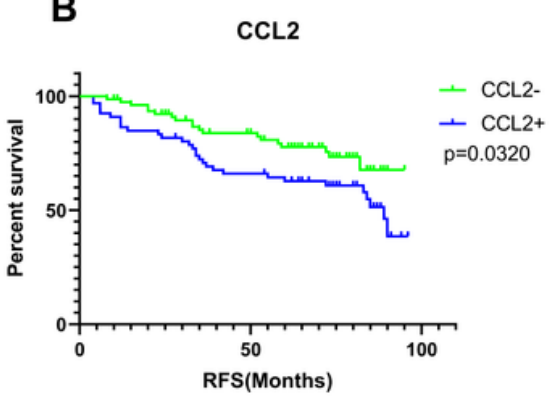

High

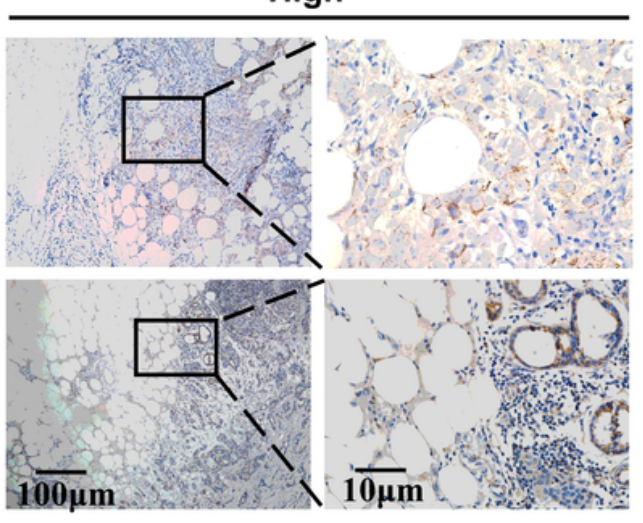

CCL5

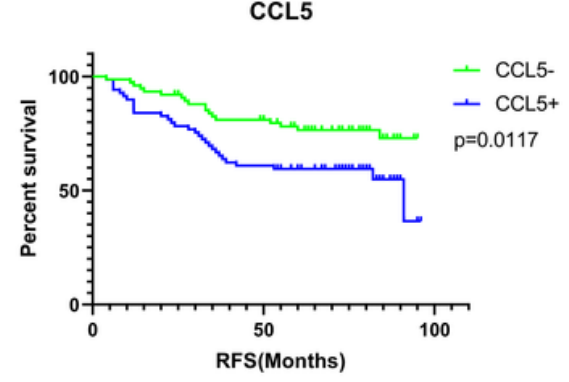

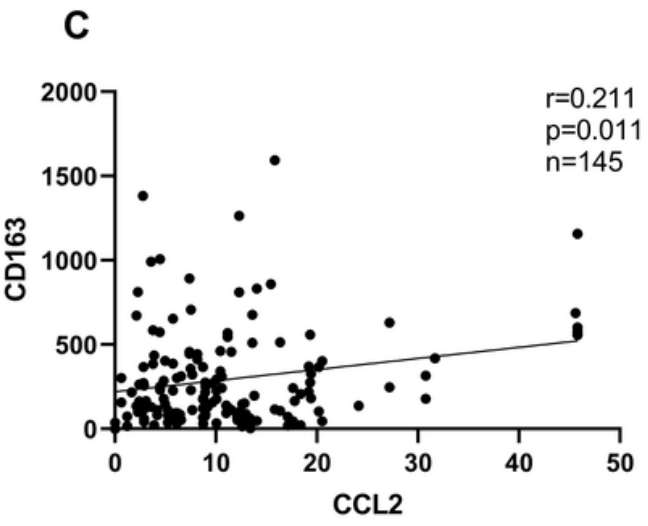

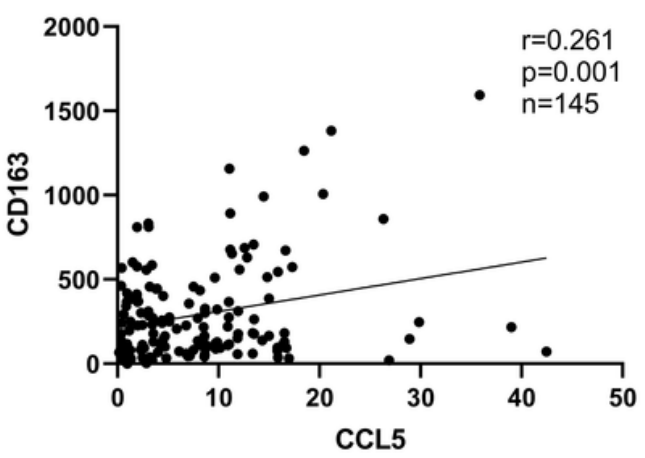

Figure 2

Chemokines CCL2 and CLL5 are associated with the infiltration of M2-like macrophages. A.

Representative immunohistochemistry staining of CCL2 and CCL5 in the specimens with breast cancer.

B. Kaplan-Meier survival analysis of patients with CCL2/CCL5-positive and -negative IHC staining, respectively. C. Correlation analyses of protein expression levels among CD163, CCL2, and CCL5. CD163 versus CCL2 (left) and CCL5 (right). 
A

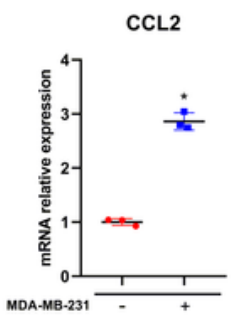

B

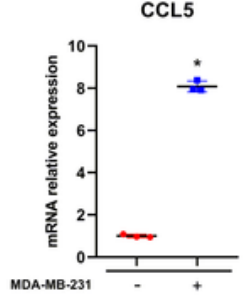

c

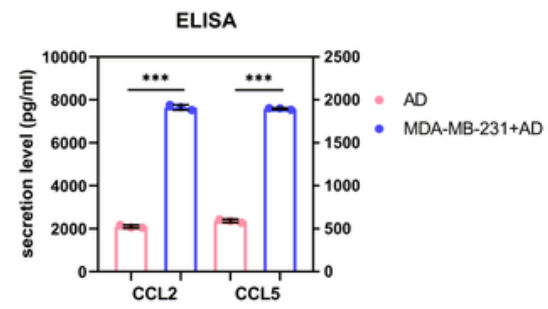

D
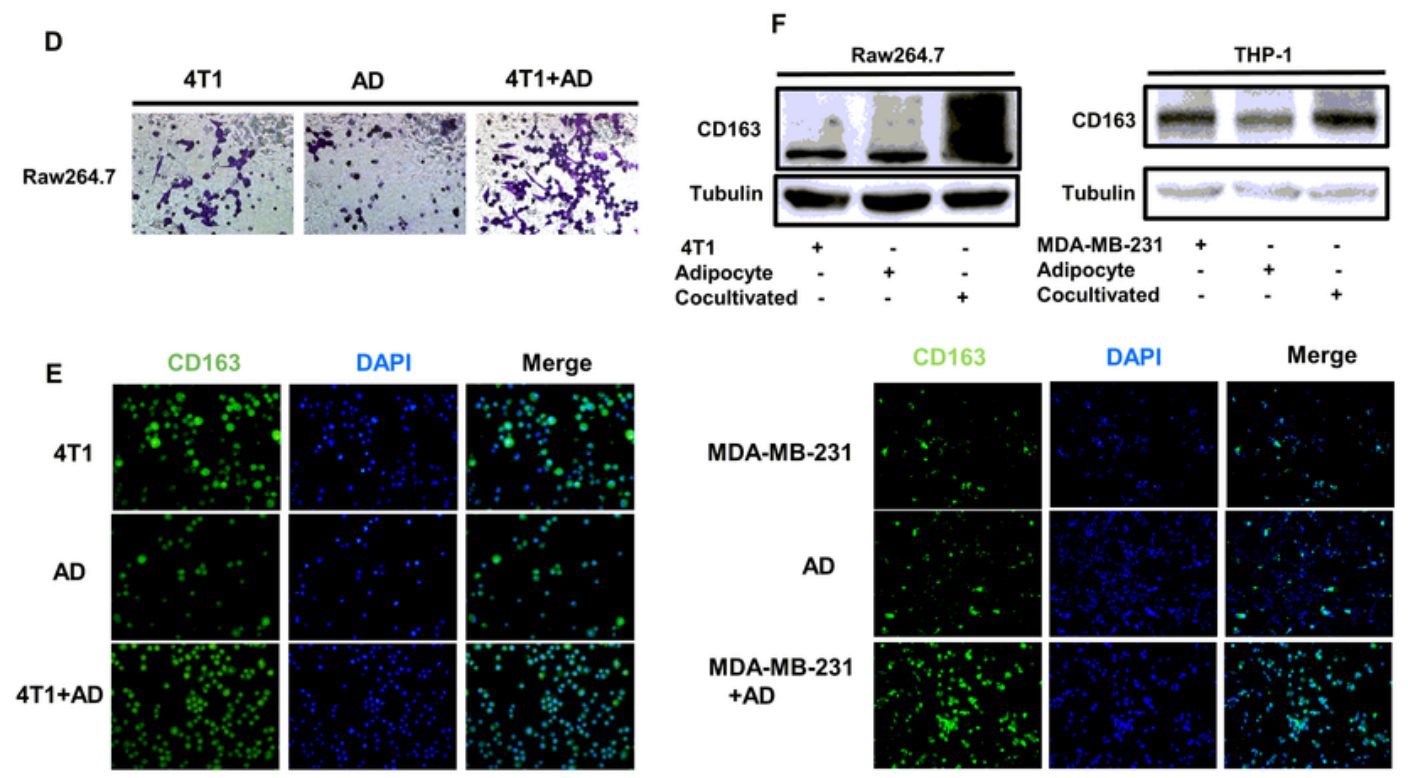

G
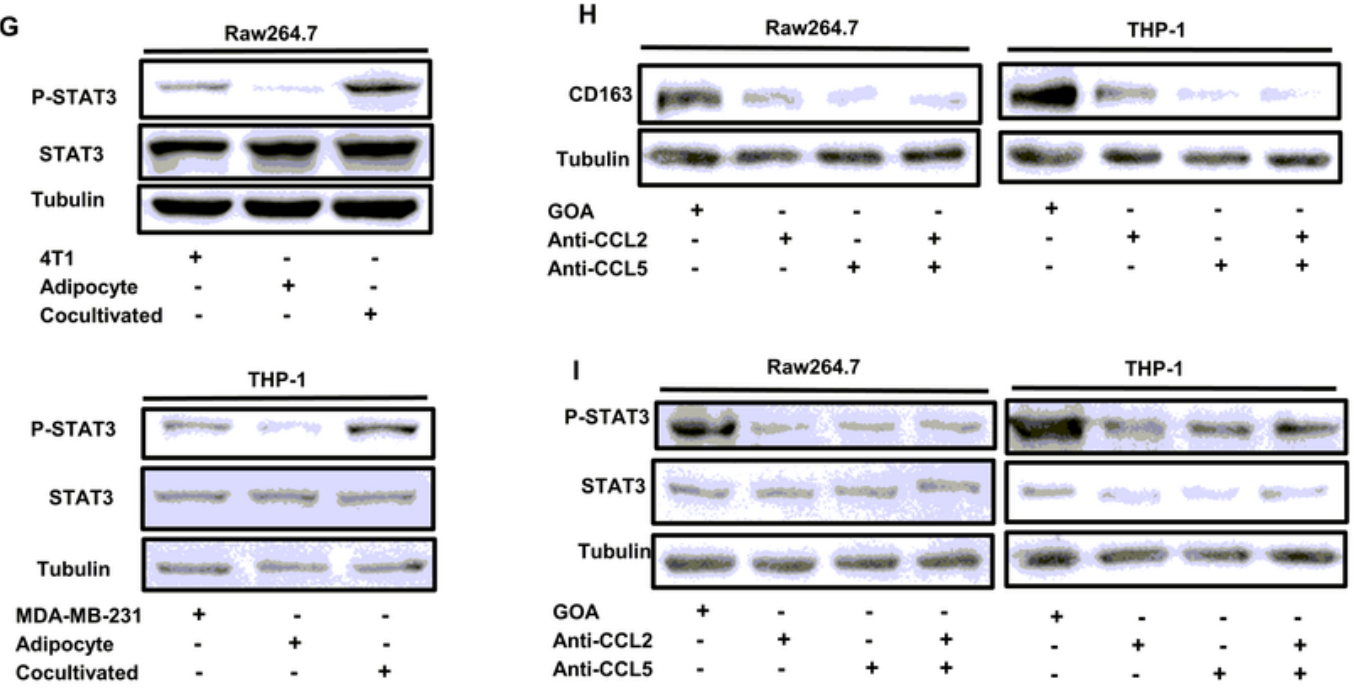

\section{Figure 3}

Chemokines CCL2 and CCL5 promoted M2-like polarization of macrophages by activating STAT3. A-B. The mRNA expression levels of CCL2 and CCL5 in mature adipocytes in the presence or absence of MDAMB-231 cells. C. The secretion levels of CCL2 and CCL5 in the media of mature adipocytes in the presence or absence of MDA-MB-231 cells. D. Macrophages were cultivated in cancer cell CM, AD-CM, or CA-CM. After $24 \mathrm{~h}$, the number of cells penetrating the membrane in Transwell invasion assays were 
analyzed. E. Immunofluorescence staining for macrophages with the treatment of 4T1/MDA-MB-231 conditioned media (CM), adipocyte-conditioned medium (AD-CM), and cancer-associated adipocyte conditioned medium (CA-CM) for 3 days. F. After macrophages were treated for 3 days, western blot was used to analyze related protein expression in different groups. G. pSTAT3 and STAT3 protein expression were analyzed by western blot in extracts from macrophages cultivated in cancer cell $C M, A D-C M$, or $C A-$ CM for 3 days. H. CA-CM was neutralized by CCL2 or CCL 5 neutralizing antibodies overnight before treating macrophages, and the control group was neutralized by Goat serum (GOA). After treatment with neutralized CA-CM for 3 days, CD163 protein expression was analyzed by western blot. I. pSTAT3 and STAT3 protein expression were analyzed by western blot in macrophages cultivated in neutralized CA-CM for 3 days. The bars represent the mean \pm SD of triplicate datapoints $(n=3) . * P<0.05$ versus control values. 
A

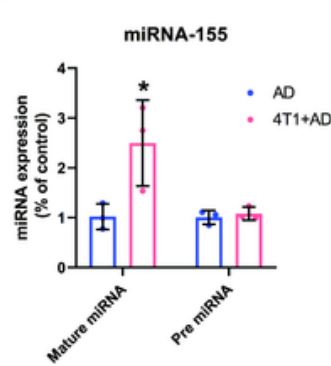

B

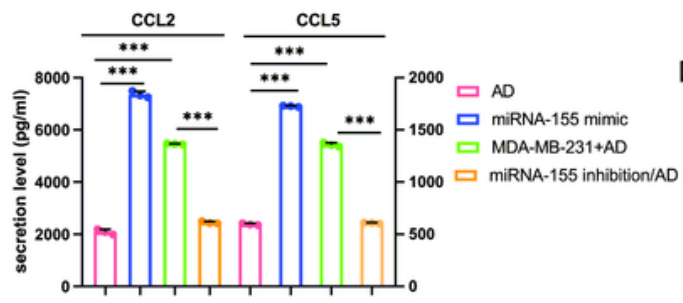

F

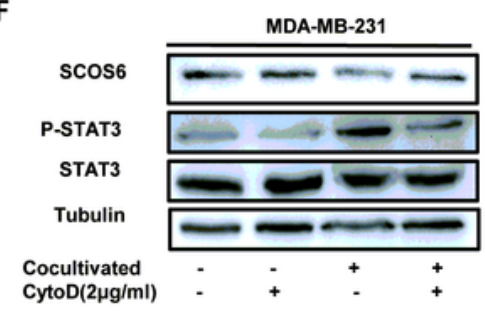

H

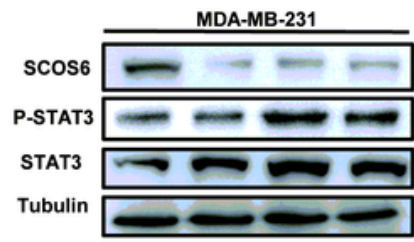

Cocultivated

miRNA-155 mimic

miRNA-155 inhibition

J

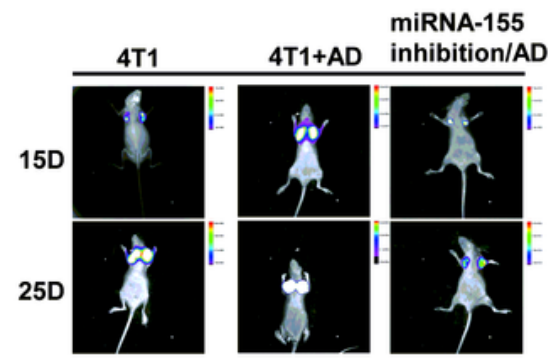

G

I
exomiRNA-155

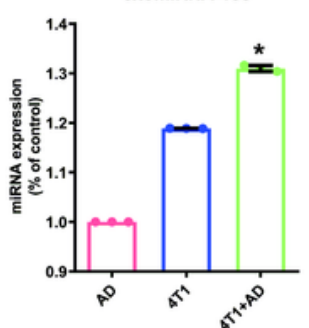

C socs6 5' CACUgUGAUUUUUAUGCAUUAAA 3' miR-155 3' UgGgGAUAGUGCUAAUCGUAAUU 5

D

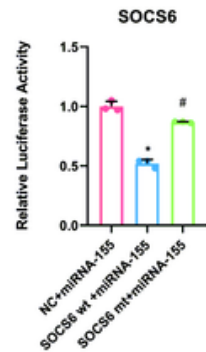

E
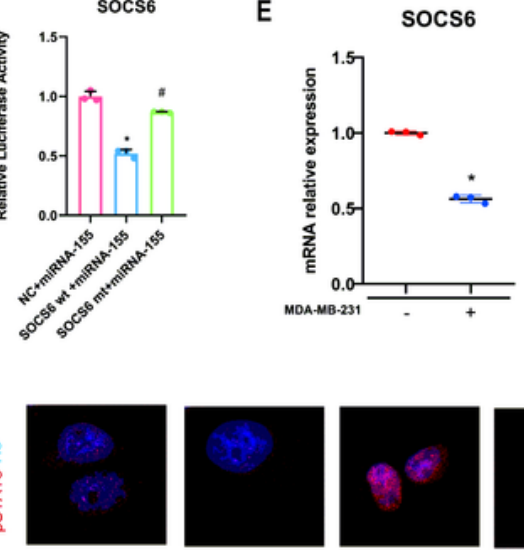

Cocultivated

CytoD $(2 \mu \mathrm{g} / \mathrm{ml})$

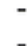

$+$

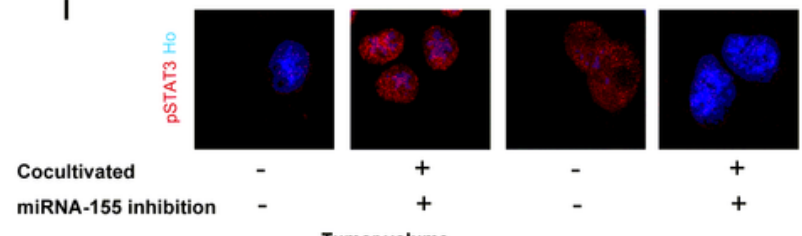

$\mathrm{K}$

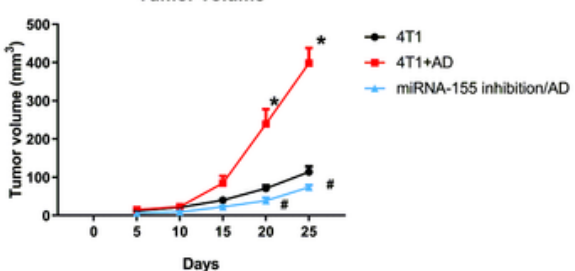

L

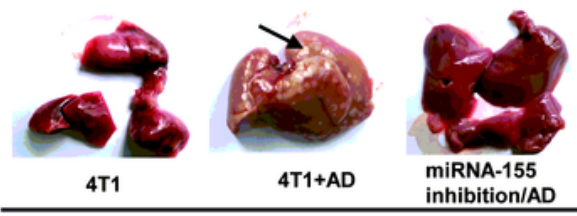

Liver

Figure 4

Tumor-derived ExomiR-155 mediates inflammatory files of adipocytes by targeting SOCS6/STAT3 signaling. A. Adipocytes were cocultivated in the presence or absence of 4T1 or MDA-MB-231 cells. After 3 days, RNA was extracted from the adipocytes and mature miRNA-155 and pre miRNA-155 were further verified by qPCR (left and middle). ExomiR-155 was also verified by qPCR (right). B. The secretion levels of CCL2 and CCL5 in different groups. C. The predicted miR-155 binding site in the 3'UTR of the SOCS6 
gene from TargetScan. D. The GV272 vector containing the 3'UTR of the target gene harbouring wild-type (wt) or mutated (mt) miRNA binding sites was transfected into HEK 293 T cells stably expressing miRNA or empty vector (as a control). Luciferase activity was analyzed at $48 \mathrm{~h}$ post-transfection, and the ratio of firefly luciferase activity to Renilla luciferase activity is shown. E. The mRNA expression levels of SOCS6 in mature adipocytes in the presence or absence of MDA-MB-231 cells. F-G. Western blot analysis of related protein expression and Immunofluorescence staining for PSTAT3 in different groups. The adipocytes in the cytochalasin D (CytoD) group were treated with CytoD (final concentration, $2 \mu \mathrm{g} / \mathrm{ml}$ ) and $20 \mu \mathrm{g}$ of exosomes purified from CA-CM. H-I. Western blot analysis of related protein expression and Immunofluorescence staining for pSTAT3 in different groups. Breast cancer cells were transfected with miR-155 inhibitor, and mature adipocytes were transfected with miR-155 mimic as the positive control. JL, miRNA-155-knockdown 4T1 cells were co-injected with mature 3T3-L1 cells into the mammary and axilla fat pads of mice. As a control, normal 4T1 cells mixed with or without adipocytes in Matrigel were inoculated into the mammary and axilla fat pads of BALB/c nude mice (5 mice in each group). Tumour size was measured every 5 days stopping at day 25. N, Macro metastatic lesions were observed in the livers (arrow). Data are presented as the mean \pm S.D. of at least three independent experiments. ${ }^{*} P<0.05$ versus control values.

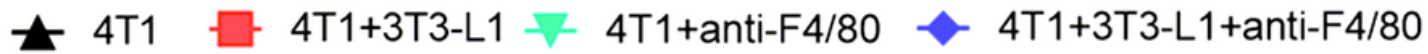

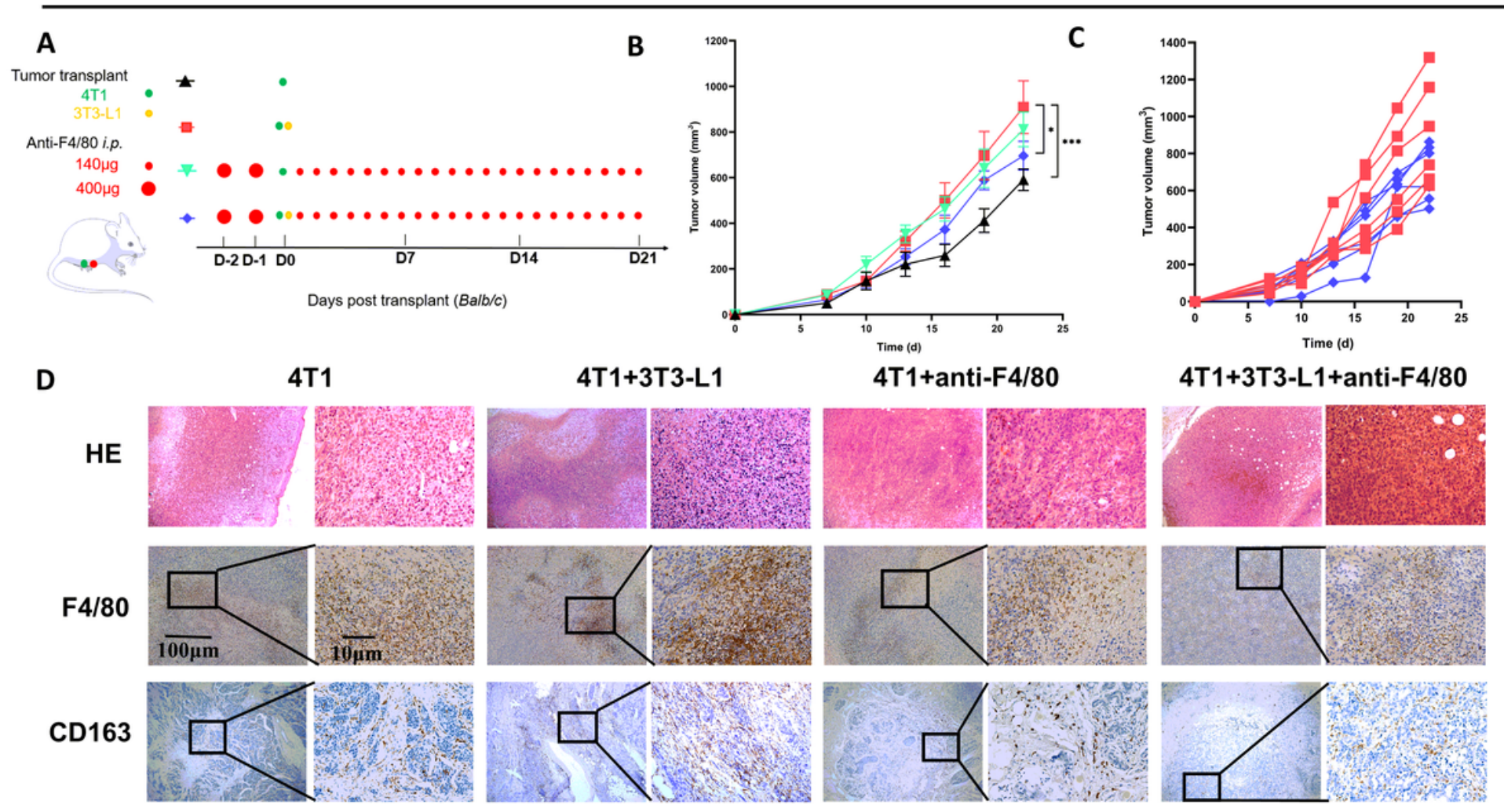

\section{Figure 5}

Depleting macrophages in adipose tissues receded adipocytes-stimulated tumor proliferation. A. $3 \times 105$ mouse breast cancer cells 4T1 or both 4T1 and mature 3T3-L1 (4:1) were injected in the mammary fat

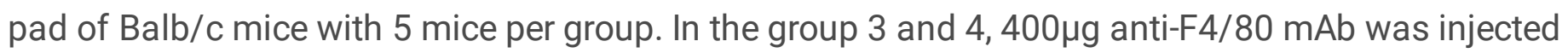


intraperitoneally (i.p.) for two days before tumor cells were injected, subsequently $140 \mu \mathrm{g}$ anti-F4/80 mAb was administered i.p. every day until the end. B. Tumor size was assessed regularly, and individual tumor growth curves of four groups. C. Individual tumor growth curves of 4T1+3T3-L1 versus 4T1+3T3-L1+antiF4/80 were depicted. Significances were tested using a type II ANOVA test. D. HE staining was performed for the histologic analysis of tumors. In addition, the infiltration of macrophages and M2-like macrophages were detected via IHC staining with anti-F4/80 and anti-CD163 antibodies, respectively.

A Cells s.c.

BMS-813160 gavage

anti-CCLs i.v.

umor monitoring

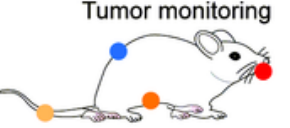

B

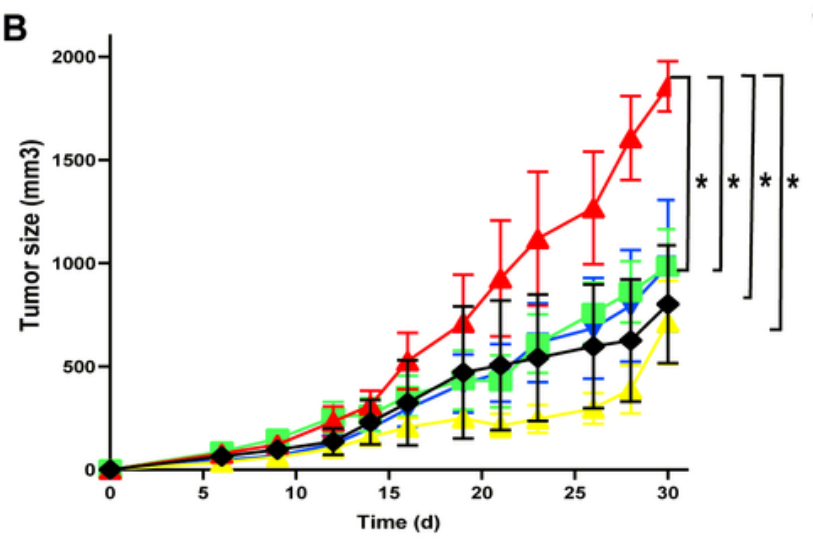

D

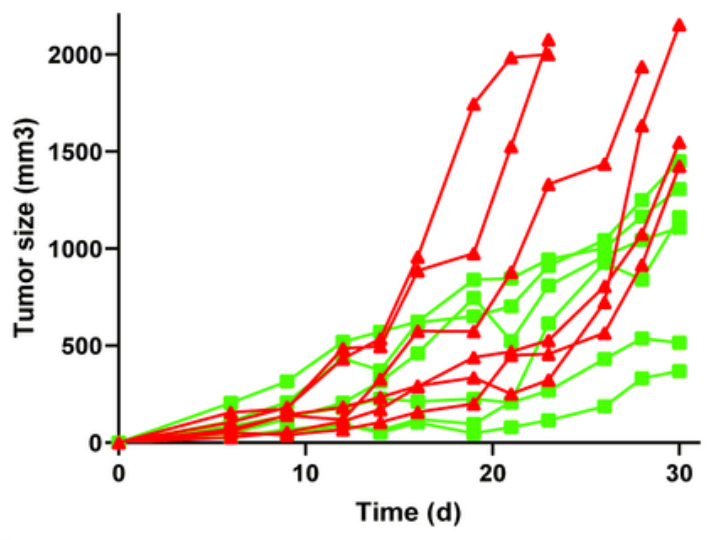

E

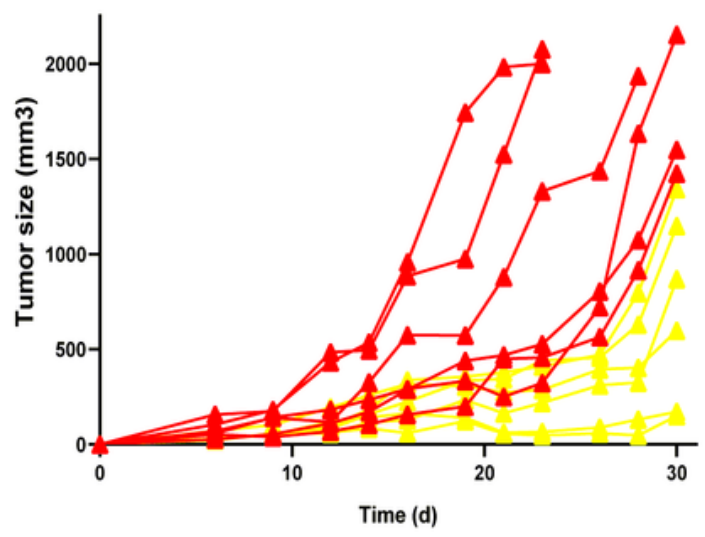

- Control

$\pm A D+A T 3$

6 AD+AT3+Stattic

- $A D+A T 3+B M S-813160$

₹ $A D+A T 3+a n t i-C C L 2 / C C L 5$

C

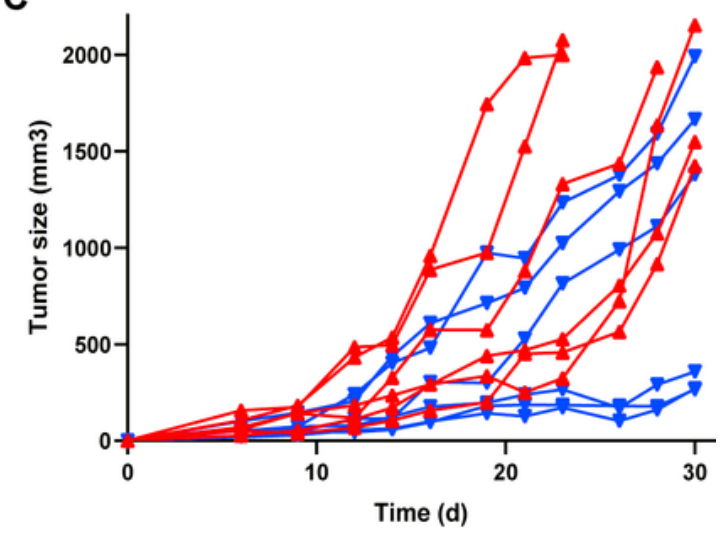

$\mathbf{F}$

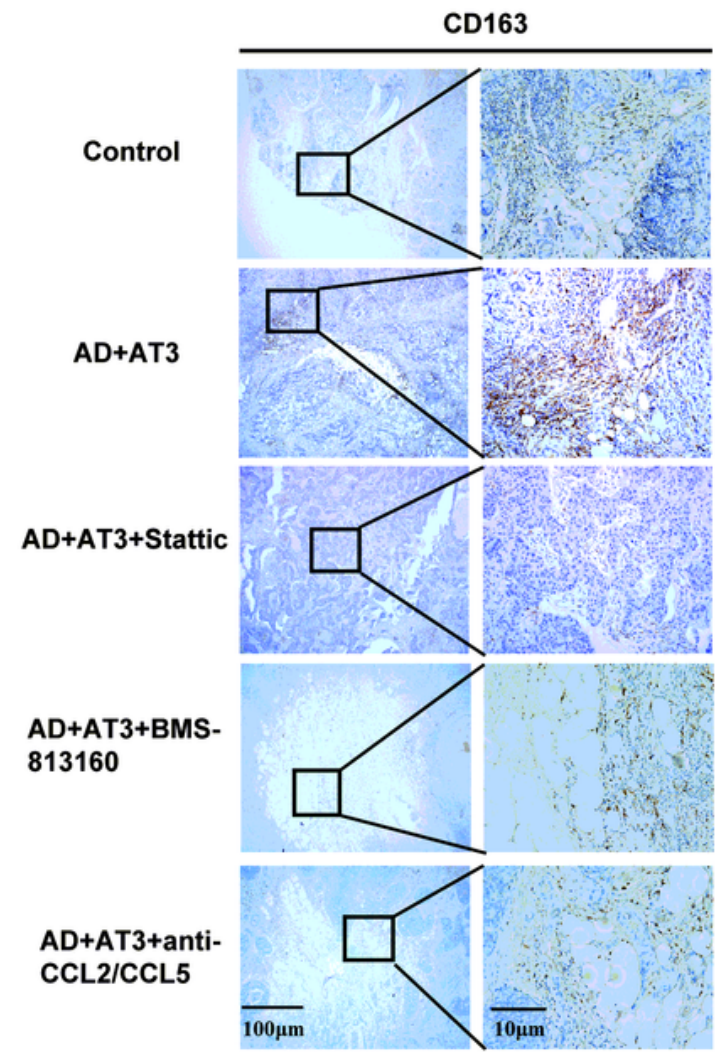

Figure 6 
Blocking the chemokines/receptors/STAT3 axis prevents breast cancer growth. A. Schematic overview of the in vivo treatment of murine mammary carcinoma AT-3 cells alone or in combination with mature adipocytes, further treated by STAT3 inhibitor Stattic, blocking antibodies against CCL2 and CCL5, or the CCR2/CCR5 antagonist BMS-813160, respectively. B. Growth kinetic of murine mammary carcinoma AT-3 cells was evolving in immunocompetent $\mathrm{C} 57 \mathrm{BI} / 6$ mice, treated as indicated in (A). $n \geq 6$ for mice in each group. Results (means \pm SD tumor growth curves) are plotted ( ${ }^{*}<<0.05, * * P<0.01$ vs. control; ns, not statistically significant; Student's t-test). C-E. Individual tumor growth curves of mice in AT-3 cells combined with mature adipocytes, respectively treated by STAT3 inhibitor Stattic, blocking antibodies against CCL2 and CCL5, or the CCR2/CCR5 antagonist BMS-813160.

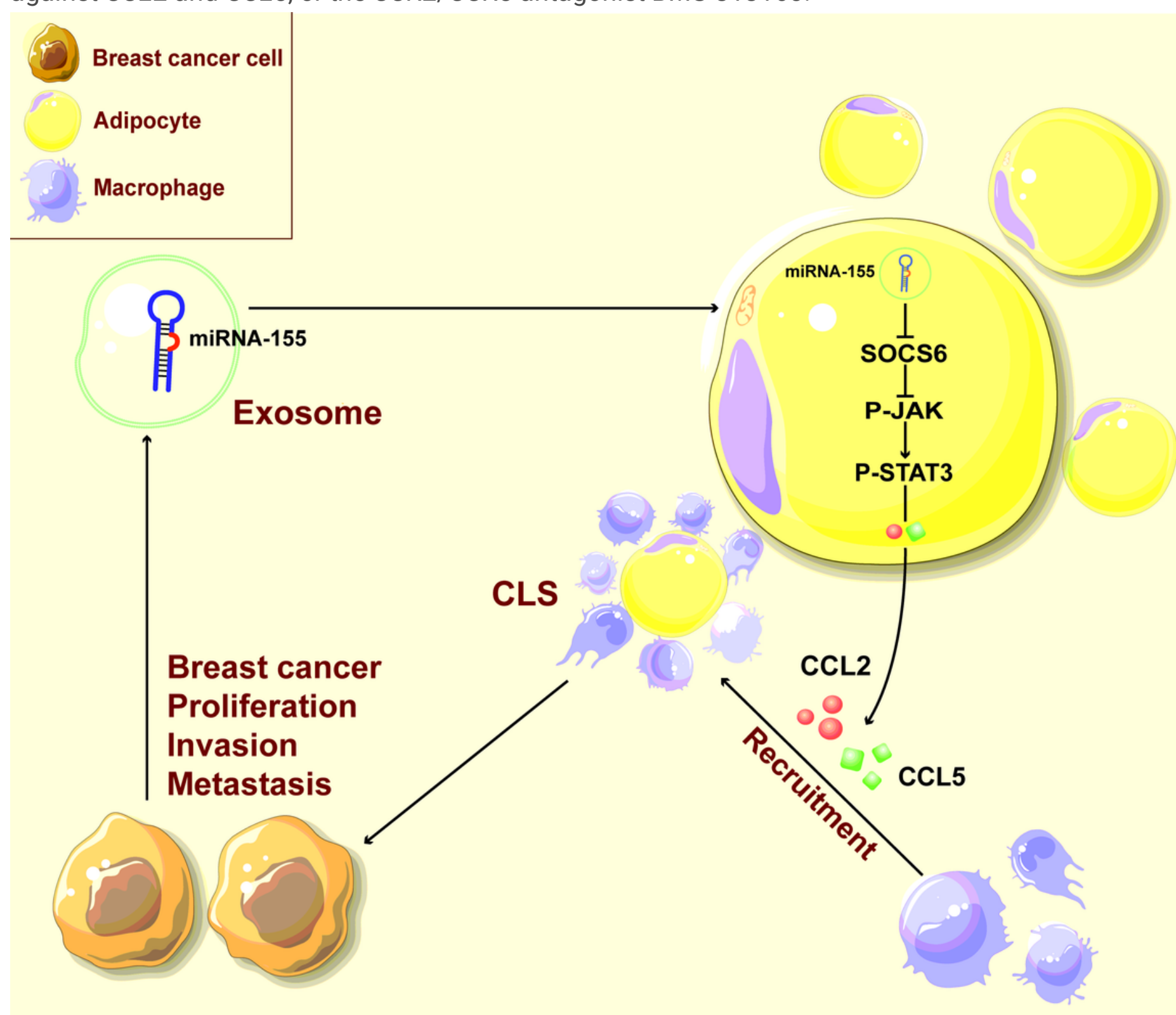

Figure 7

The proposed molecular model by which miR-155 facilitates the tumor microenvironment. 


\section{Supplementary Files}

This is a list of supplementary files associated with this preprint. Click to download.

- FigureS1.tif

- Figures2.tif

- Figures3.tif

- FigureS4.tif

- Figures5.tif

- Tables1.docx

- TableS2.docx

- TableS3.docx

- Tables4.docx 